\title{
On Deterministic Online Scheduling: Major Considerations, Paradoxes and Remedies
}

\author{
Dhruv Gupta, Christos T. Maravelias* \\ Department of Chemical and Biological Engineering, University of Wisconsin-Madison \\ 1415 Engineering Drive, Madison, WI 53706, USA
}

\begin{abstract}
Despite research in the area, the relationship between the (open-loop) optimization problem and the quality of the (closed-loop) implemented schedule is poorly understood. Accordingly, we first show that open-loop and closed-loop scheduling are two different problems, even in the deterministic case. Thereafter, we investigate attributes of the open-loop problem and the rescheduling algorithm that affect closed-loop schedule quality. We find that it is important to reschedule periodically even when there are no "trigger" events. We show that solving the open-loop problem suboptimaly does not lead to poor closed-loop solutions; instead, suboptimal solutions are corrected through feedback. We also observe that there exist thresholds for rescheduling frequency and moving horizon length, operating outside of which leads to substantial performance deterioration. Fourth, we show that the design attributes work in conjunction, and hence studying them simultaneously is important. Finally, we explore objective function modifications and constraint addition as methods to improve performance.

Keywords: Chemical production scheduling; Rescheduling; Mixed-integer programming; Closed-loop solution.
\end{abstract}

*Corresponding author: christos.maravelias@wisc.edu 


\section{Introduction}

Process industries employ a complex chain of operations (processes) which compete for limited resources. Hence a resource allocation problem arises wherein decisions with regards to the start time of operations, how much to process, the equipment to utilize and several associated decisions need to be taken on a repetitive basis.

Traditionally, schedules have been constructed by experienced scheduling personnel (schedulers) using spreadsheets or heuristics-based software. In the last two decades however, there has been an increasing thrust towards using advanced computational tools to achieve more profitable schedules. Hence, working with scheduling models to optimally plan operations has become an important problem in process systems engineering (Shobrys and White, 2002; Kelly and Mann, 2003a,b; Harjunkoski et al., 2014). Although the process systems engineering (PSE) community has worked on building accurate models and better solution methods, the aspect of rescheduling has received limited attention. Rescheduling has been emphasized in some works (Cott and Macchietto, 1989; Kanakamedala et al., 1994; Huercio et al., 1995; Rodrigues et al., 1996; Vin and Ierapetritou, 2000), but in most cases scheduling is still thought to be a static open loop problem wherein if rescheduling is carried out, the emphasis is only on restoring feasibility or optimality to the current static schedule. Quantifying the quality of the implemented schedule obtained by rescheduling has not been addressed. Accordingly, the goal of this paper is to study how the open-loop optimization problem impacts the quality of the implemented (closed-loop) schedule.

In a dynamic environment multiple disturbances such as task delays, yield losses, unit breakdowns or rush order arrivals can render a previously computed schedule suboptimal or even infeasible. In addition, Subramanian et al. (2012) emphasized that rescheduling also needs to be performed due to advancement of the scheduling horizon over which the schedule is computed even when there are no disturbances. Rescheduling has been demonstrated to lead to lower inventory accumulation in supply chains (Subramanian et al., 2014).

In this work, we first and foremost show that open-loop and closed-loop scheduling are 
two different problems, even in the deterministic case, when no uncertainty is present. Thereafter, we investigate how the design of the open-loop optimization problem affects the quality of the resulting implemented closed-loop schedule. The design attributes we study are scheduling horizon length, rescheduling frequency and optimality gap of each open-loop optimization. We choose illustrative process networks and extensively study combinations of the aforementioned attributes over a reasonably exhaustive set of short term demand patterns, production load, and scheduling objectives. From these test cases, we identify trends, and problem instance characteristics which can facilitate in carefully choosing the three attributes.

The paper is structured as follows: In section 2, we present background on chemical production scheduling and discuss past work on rescheduling. In section 3, we discuss various design considerations in closed-loop scheduling through motivating examples. In section 4, we describe the problem library we use. We then present results in section 5, followed by, a discussion in section 6 . Thereafter, we present several case studies in section 7 , and then provide conclusions in section 8. We use lower case Latin characters for indices, uppercase bold Latin characters for sets, lowercase Greek characters for parameters, and uppercase Latin characters for variables.

\section{Background}

\subsection{Previous research}

A schedule can become suboptimal or even infeasible due to, for example, a disruptive event or a change in resource availability. The revision of an existing schedule in response to disruptions or changes is termed as reactive scheduling or rescheduling. When the focus of rescheduling is only on a schedule for a fixed horizon, it results in a shrinking-horizon problem. On the other hand, when the current schedule is possibly revised, and also additional decisions are made for the time ahead, it naturally results in a moving-horizon (or rollinghorizon) problem. Past works have focused primarily on shrinking-horizon approaches. 
Kanakamedala et al. (1994) investigated a bi-level least impact heuristic for rescheduling. Huercio et al. (1995) presented a heuristic for rescheduling through task start time shifting and unit reallocation. Bassett et al. (1997) formulated a Monte Carlo framework for determining due-time reliability when processing times and equipment reliability were uncertain. Using this Monte Carlo framework Honkomp et al. (1999) developed a simulator to evaluate and compare rescheduling policies for handling processing time uncertainty. Méndez and Cerdá (2003) discussed a user controlled flexible MILP systematic tool to repair schedules by limited reallocation and reordering of operations in an iterative fashion. Janak et al. (2006) proposed identifying and fixing of future production unaffected by realized disturbances when carrying out rescheduling. Relvas et al. (2007) applied rescheduling to scheduling of multiproduct pipelines. Novas and Henning $(2010,2012)$ proposed repair-based and rolling-horizon based rescheduling algorithms using constraint programming techniques.

Munawar and Gudi (2005) proposed an integrated multilevel control theoretic framework for integration of planning and scheduling. Cui and Engell (2010) demonstrated application of moving horizon scheduling to an expandable polystyrene plant. Zhuge and Ierapetritou (2012) presented a closed-loop strategy for integrated scheduling and control and emphasized that rescheduling should be performed to take advantage of any favorable disturbance to process rather than trying to reject it to maintain the incumbent schedule. Nie et al. (2014) proposed a resource-task network based state-space model for rescheduling. Lindholm et al. (2013) formulated a scheduling model for continuous production sites and solved it every day in a receding horizon fashion given updates of actual production each day and incoming orders. Kopanos and Pistikopoulos (2014) implemented rescheduling using state-space model and multi-parametric programming in a rolling horizon framework. Du et al. (2015) discussed a time-scale bridging approach for closed-loop scheduling and control.

Methods for optimization under uncertainty have been proposed to address scheduling disturbances, including robust optimization (Yu and Li, 2000; Lin et al., 2004; Bonfill et al., 2005; Janak et al., 2007), stochastic optimization (Bonfill et al., 2004; Balasubramanian 
and Grossmann, 2004; Sand and Engell, 2004), and fuzzy programming approaches (Balasubramanian and Grossmann, 2003; Wang, 2004; Petrovic and Duenas, 2006). In addition, multi-parametric programming has been used for proactive scheduling (Ryu et al., 2007; Li and Ierapetritou, 2008b). Li and Ierapetritou (2008a) provide a good review on scheduling under uncertainty.

\subsection{Chemical production scheduling}

\subsubsection{General problem statement}

The general problem can be stated as follows. Given:

(i) Production facility data (e.g., unit capacities and connectivity);

(ii) Production recipes (e.g., processing times and mixing rules);

(iii) Production costs (e.g., material holding costs);

(iv) Material availability (e.g., raw materials delivery amounts and dates);

(v) Resource availability (e.g., maintenance schedule and utility levels); and

(vi) Production targets or orders with due-times;

scheduling seeks to find

(i) Selection and sizing of batches,

(ii) Assignment of these batches to units,

(iii) Timing of these batches;

so as to meet production targets at minimum cost, or to maximize profit if production beyond given target is allowed.

In general, several processing characteristics and constraints could be present such as sequence dependent changeovers, setup times, storage constraints, time-varying utility costs 
etc. The objective could be, in addition to minimization of cost or maximization of profit, minimization of makespan, minimization of earliness or any other suitable objective for the specific application (Méndez et al., 2006; Harjunkoski et al., 2014).

\subsubsection{Problem representation}

An abstract framework is required to represent the different elements of a production facility and the associated production recipe before the corresponding scheduling problem can be defined and solved. To this end, Kondili et al. (1993) proposed the state task network (STN) representation for facilities wherein materials (states) are transformed through tasks. These tasks can be carried out on units (equipment) and they may also require utilities. In addition, Pantelides (1994) proposed a unified framework, called resource task network (RTN), for representing scheduling problems where materials, equipment and utilities are all treated at par as resources.

The STN representation primarily comprises of tasks $i \in \mathbf{I}$, units $j \in \mathbf{J}$, and materials $k \in \mathbf{K}$ which are further elucidated as follows (see Fig. 1 for an example):

(i) A set of tasks $i \in \mathbf{I}$ : set of tasks producing/consuming material $k$ are denoted by $\mathbf{I}_{k}^{+} / \mathbf{I}_{k}^{-}$; task $i$ consumes/produces material $k, \rho_{i k}$ mass fraction of its batch-size $\left(\rho_{i k}<0\right.$ for consumption and $\rho_{i k}>0$ for production); the processing time of task $i$ is $\tau_{i}$;

(ii) A set of equipment units $j \in \mathbf{J}$ : the subset of tasks that can be carried out on unit $j$ is denoted by $\mathbf{I}_{j}$; on any given unit only one task can be performed at a time with its batch-size between lower $\left(\beta_{i j}^{M I N}\right)$ and upper capacities $\left(\beta_{i j}^{M A X}\right)$; the associated fixed and proportional production costs of carrying out task $i$ on unit $j$ are $\alpha_{i j}^{F}$ and $\alpha_{i j}^{P}$ respectively;

(iii) A set of materials $k \in \mathbf{K}$ : feed, intermediate and product materials are denoted by $k \in \mathbf{K}^{\mathbf{F}} / \mathbf{K}^{\mathbf{I}} / \mathbf{K}^{\mathbf{P}}$; there are possible incoming deliveries and outgoing orders at certain times for select materials; the selling price, inventory cost and backlog cost of material $k$ are $\pi_{k}, \pi_{k}^{I N V}$ and $\pi_{k}^{B O}$, respectively. 


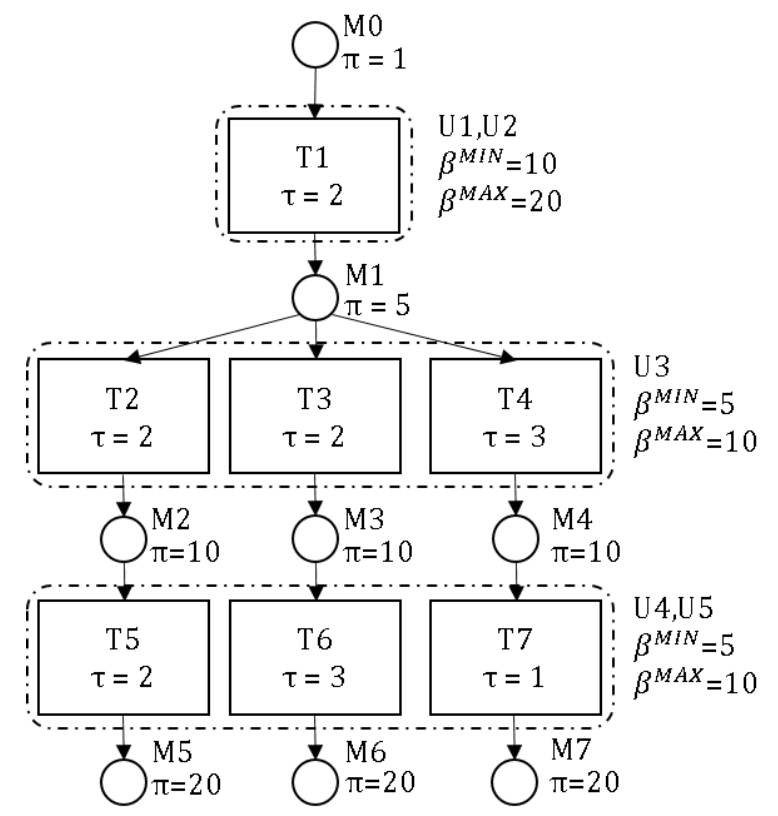

Figure 1: State task network (STN) representation for a process network (PN-1) comprising of 8 material nodes (circular) labeled M0-M7 and 7 task nodes (rectangular) labeled T1-T7. Arcs connect task nodes with corresponding input/output material nodes. Tasks can be carried out in several compatible units and can draw resources from shared resource pools, for example steam, electricity and manpower. Task-unit mapping and task batch-size capacities $\left(\beta^{M I N} / \beta^{M A X}\right)$ are shown here, but can also be tabulated separately. Material prices $(\pi)$ are shown adjacent to material nodes.

\subsubsection{Models}

Maravelias (2012) proposed a classification of models on the basis of (i) optimization decisions, (ii) modeling elements, and (iii) modeling of time. Time-grid based models can be classified as continuous time or discrete time-grid models. Although discretizing the time-grid introduces an approximation error, discrete time models have several advantages over continuous time grid models. For example, accounting for inventory or utility costs in continuous time models introduces nonlinearities but not so in discrete time-grid models (Velez and Maravelias, 2013c). Furthermore, Sundaramoorthy and Maravelias (2011) showed that discrete time models are, in general, at least as effective as continuous time models, and in fact are better suited for large scale problems with several additional processing features.

In the subsections to follow we present a discrete time STN model and a state-space model, which we use in our study. Although for our study we employ this specific model, the framework and analysis presented are applicable to all discrete-time models and can be 
extended to continuous-time models.

\subsection{Scheduling MILP model}

The discrete time STN MILP scheduling model (modified from Shah et al. (1993)) com-

prises of Eqs. 1 through 6. Time is represented by index $t \in \mathbf{T}$. Variable $W_{i j t} \in\{0,1\}$ denotes the start binary of task $i$ in unit $j$ at time $t$ and variable $B_{i j t} \in\left[\beta_{i j}^{M I N}, \beta_{i j}^{M A X}\right]$ denotes its batch-size. The assignment constraint (Eq. 1) ensures that a new task can be initiated on a unit only if the unit is not already executing a task.

$$
\sum_{i \in \mathbf{I}_{j}} W_{i j t}+\sum_{i \in \mathbf{I}_{j}} \sum_{n=1}^{\tau_{i}-1} W_{i j(t-n)} \leq 1 \quad \forall j, t
$$

The batch-size constraint (Eq. 2) ensures that the batch-size of a task is within its acceptable capacity range only when the task is executed.

$$
\beta_{i j}^{M I N} W_{i j t} \leq B_{i j t} \leq \beta_{i j}^{M A X} W_{i j t} \forall i, j, t
$$

The inventory of material $k$ during time-period $(t-1, t], S_{k t}$, is calculated as a balance of consumption/production and incoming/outgoing shipments,

$$
\begin{gathered}
S_{k(t+1)=S_{k t}+} \sum_{j} \sum_{i \in \mathbf{I}_{j} \cap \mathbf{I}_{k}^{+}} \rho_{i k} B_{i j\left(t-\tau_{i}\right)}+\sum_{j} \sum_{i \in \mathbf{I}_{j} \cap \mathbf{I}_{k}^{-}} \rho_{i k} B_{i j t} \\
-V_{k t}^{D}-V_{k t}^{S}+\zeta_{k t} \quad \forall k, t
\end{gathered}
$$

where $\zeta_{k t}$ denotes incoming shipments of material $k$ and variables $V_{k t}^{D}, V_{k t}^{S}$ refer to outgoing shipments for meeting orders and excess-sales respectively. In the cost minimization problem, shipment variables for excess-sales $\left(V_{k t}^{S}\right)$ are fixed to zero.

Equation 4 couples the outgoing shipment variable $V_{k t}^{D}$ with demand, $\xi_{k t}$, for material $k$ at time $t$. Backlog variables $B O_{k t}$ serve as slack variables denoting unmet (pending) orders during time-period $(t-1, t]$ and are penalized in the cost minimization objective function. 
In the profit maximization problem, since all orders must be met, backlog variables $\left(B O_{k t}\right)$ are fixed to zero.

$$
B O_{k(t+1)}=B O_{k t}+\xi_{k t}-V_{k t}^{D} \forall k, t
$$

Finally the domain of all the variables is restricted via Eq. 5.

$$
W_{i j t} \in\{0,1\} ; B_{i j t}, V_{k t}^{D}, V_{k t}^{S}, S_{k t}, B O_{k t} \geq 0
$$

The scheduling objective for cost minimization and profit maximization is defined by Eqs. 6a and $6 \mathrm{~b}$ respectively.

$$
\begin{gathered}
z_{\mathrm{cost}}=\min \sum_{k} \sum_{t} \pi_{k}^{I N V} S_{k t}+\sum_{k} \sum_{t} \pi_{k}^{B O} B O_{k t} \\
+\sum_{j} \sum_{i \in \mathbf{I}_{j}} \sum_{t} \alpha_{i j}^{F} W_{i j t}+\sum_{j} \sum_{i \in \mathbf{I}_{j}} \sum_{t} \alpha_{i j}^{P} B_{i j t} \\
z_{\text {profit }}=\max \sum_{k} \sum_{t} \pi_{k} V_{k t}^{S}-\sum_{j} \sum_{i \in \mathbf{I}_{j}} \sum_{t} \alpha_{i j}^{F} W_{i j t} \\
-\sum_{j} \sum_{i \in \mathbf{I}_{j}} \sum_{t} \alpha_{i j}^{P} B_{i j t}
\end{gathered}
$$

\subsection{State-space model}

Subramanian et al. (2012) proposed a state-space model, for a general chemical production scheduling problem. Analogous to state-space representation in process control (Rawlings and Mayne, 2009), they identified inputs, states and disturbances. The states are inventory $\left(S_{k t}\right)$ and backlogs $\left(B O_{k t}\right)$; the inputs are start binary of tasks $\left(W_{i j t}\right)$, tasks batch-size $\left(B_{i j t}\right)$, and the shipments $\left(V_{k t}^{D}, V_{k t}^{S}\right)$; and disturbances are demand orders $\left(\xi_{k t}\right)$, 
task delays, material handling losses and unit breakdowns. In this work, we only consider the demand orders disturbance. The inputs, start binary of tasks $\left(W_{i j t}\right)$ and corresponding batch-sizes $\left(B_{i j t}\right)$, have a delayed effect on future states, hence they are lifted.

The original start binary, $W_{i j t}$, and batch-size, $B_{i j t}$, in the STN model are represented by variables $\bar{W}_{i j t}^{0}$ and $\bar{B}_{i j t}^{0}$, respectively, , in the state-space model. Lifting of these past input variables is achieved through Eqs. 7 and 8. Binary variable $\bar{W}_{i j t}^{n}$ is 1 if at time $t$, task $i$ has been under execution on unit $j$ for $n$ time-periods. When the task starts, $n$ is zero $(n=0)$, and when the task is finishes, $n$ equals the processing time of the task $\left(n=\tau_{i}\right)$. Equations 1-4 are rewritten in terms of lifted variables as Eqs. 9-12 for the state-space model.

$$
\begin{gathered}
\bar{W}_{i j(t+1)}^{n}=\bar{W}_{i j t}^{n-1} \quad \forall j, i \in I_{j}, t, n \in\left\{1,2, \ldots \tau_{i}\right\} \\
\bar{B}_{i j(t+1)}^{n}=\bar{B}_{i j t}^{n-1} \quad \forall j, i \in I_{j}, t, n \in\left\{1,2, \ldots \tau_{i}\right\} \\
\sum_{i \in \mathbf{I}_{j}} \bar{W}_{i j t}^{0}+\sum_{i \in \mathbf{I}_{j}} \sum_{n=1}^{\tau_{i}-1} \bar{W}_{i j t}^{n} \leq 1 \quad \forall j, t \\
\beta_{i j}^{M I N} \bar{W}_{i j t}^{0} \leq \bar{B}_{i j t}^{0} \leq \beta_{i j}^{M A X} \bar{W}_{i j t}^{0} \forall i, j, t \\
S_{k(t+1)}=S_{k t}+\sum_{j} \sum_{i \in \mathbf{I}_{\mathbf{j}} \cap \mathbf{I}_{\mathbf{k}}^{+}} \rho_{i k} \bar{B}_{i j t}^{\tau_{i}}+\sum_{j} \sum_{i \in \mathbf{I}_{\mathbf{j}} \cap \mathbf{I}_{\mathbf{k}}^{-}} \rho_{i k} \bar{B}_{i j t}^{0} \\
-V_{k t}^{D}-V_{k t}^{S}+\zeta_{k t} \forall k, t \\
B O_{k(t+1)}=B O_{k t}+\xi_{k t}-V_{k t}^{D} \forall k, t \\
\forall k, t
\end{gathered}
$$

The domain for all variables is restricted through Eq. 13. 


$$
\bar{W}_{i j t}^{n} \in\{0,1\} ; \bar{B}_{i j t}^{n}, V_{k t}^{D}, V_{k t}^{S}, S_{k t}, B O_{k t} \geq 0
$$

The cost minimization and profit maximization objective are defined by Eqs. 14a and 14b respectively.

$$
\begin{aligned}
z_{\text {cost }}=\min & \sum_{k} \sum_{t} \pi_{k}^{I N V} S_{k t}+\sum_{k} \sum_{t} \pi_{k}^{B O} B O_{k t} \\
& +\sum_{j} \sum_{i \in \mathbf{I}_{j}} \sum_{t} \alpha_{i j}^{F} \bar{W}_{i j t}^{0}+\sum_{j} \sum_{i \in \mathbf{I}_{j}} \sum_{t} \alpha_{i j}^{P} \bar{B}_{i j t}^{0} \\
z_{\text {profit }}=\max & \sum_{k} \sum_{t} \pi_{k} V_{k t}^{S}-\sum_{j} \sum_{i \in \mathbf{I}_{j}} \sum_{t} \alpha_{i j}^{F} \bar{W}_{i j t}^{0} \\
& -\sum_{j} \sum_{i \in \mathbf{I}_{j}} \sum_{t} \alpha_{i j}^{P} \bar{B}_{i j t}^{0}
\end{aligned}
$$

Hence, Eqs. 7 through 14 constitute the state-space scheduling model.

Although the aforementioned reformulations (Eqs. 7 and 8) for lifting past input variables increase model size, computations are not slower than the original model because solver preprocessors are able to reduce the model size through constraint and variable elimination. The state-space formulation poses scheduling in the form of linear evolution equations making it convenient to implement closed-loop scheduling (Sec. 2.6).

\subsection{Solution of MILP models}

\subsubsection{Termination criteria and quality of solution}

MILPs are typically solved using a branch and bound scheme (Wolsey, 1998). A metric, for assessing the optimality status of the instance being solved, is the relative estimated optimality gap. It refers to the gap, between the best bound on the optimal objective value i.e. the best bound of the linear-programming relaxation (LP-relaxation) of unpruned nodes, 
$Z_{B B}^{L P}$, and the objective value of the current best integer (IP) solution, $Z^{I P}$. It is calculated using the formula:

$$
\text { Relative Estimated Gap }=\frac{\left|Z_{B B}^{L P}-Z^{I P}\right|}{Z^{I P}+\epsilon}
$$

where $\epsilon$ is a very small number (here $1 \times 10^{-10}$ ). When the relative estimated gap is used as a termination criterion, we refer to it as OPTCR. The value of OPTCR can be set to any fractional value between 0 and 1, with 0 denoting terminating the search only on having found proven optimal integer solution and 1 denoting terminating at the first found feasible integer solution.

In addition to the relative estimated gap, there are several other metrics which although cannot be used as termination criteria, they are useful in better understanding the solution process once it has been completed. The two metrics we use here are relative true gap and integrality gap. Relative true gap refers to the relative gap between the current best integer solution objective, $Z^{I P}$, and the optimal integer solution objective, $Z^{*}$. It is calculated using the following formula:

$$
\text { Relative True Gap }=\frac{\left|Z^{I P}-Z^{*}\right|}{Z^{*}+\epsilon}
$$

Integrality gap refers to the relative gap between, the optimal objective, $Z_{L P}^{*}$, of the LPrelaxation, and the optimal objective, $Z^{*}$, of the MILP model:

$$
\text { Integrality Gap }=\frac{\left|Z_{L P}^{*}-Z^{*}\right|}{Z^{*}+\epsilon}
$$

\subsubsection{Solution methods}

The combinatorial nature of MILP scheduling models, makes it challenging to obtain optimal solutions. A number of works have contributed, towards making the solution of discrete time-grid based scheduling models more amenable, through the use of parallel computing (Subrahmanyam et al., 1996; Velez and Maravelias, 2013a), decomposition algorithms (Bassett et al., 1996; Kelly and Zyngier, 2008), model reformulations (Yee and Shah, 1998; Velez and Maravelias, 2013c) and pre-processing algorithms (Velez et al., 2013; Merchan and 
Maravelias, 2016). In addition, (Velez and Maravelias, 2013b, 2015) formulated nonuniformmulti-grid models which retain optimal solution while decreasing problem size.

\subsection{Closed-loop scheduling}

The term rescheduling has been used to refer to the corrective action taken to respond to disturbances (e.g., new order arrival) or an event that renders the current schedule infeasible (e.g., unit breakdown). It is thought to be performed on an "as needed" basis. In this paper, we argue that rescheduling should be performed regularly, possibly at a predetermined frequency, even if no trigger event occurs. We will refer to this periodic rescheduling as "online scheduling". Online scheduling could employ heuristics (e.g. dispatching rules) or may require solving optimization problems. We will study online scheduling, where optimization problems are solved repetitively, in a moving horizon setting while incorporating feedback (closed-loop scheduling). Henceforth in this work, we use the terms online scheduling and closed-loop scheduling interchangeably. In addition, we use the terms closed-loop schedule and closed-loop solution to mean the same.

We carry out closed-loop scheduling by solving the state-space MILP scheduling problem (Sec. 2.4) at each time step, to yield current and future scheduling decisions. As shown in Fig. 2, when the current decisions are implemented, the horizon is moved forward by a time step and the procedure is repeated. This is analogous to how model predictive control (MPC) is implemented. As opposed to full rescheduling, partial rescheduling can also be carried out by fixing some future decisions and only computing remainder of the decisions in the scheduling horizon.

The scheduling horizon is associated with the open-loop problem. The open-loop problem is the optimization problem that a scheduler would solve on a day to day basis to yield current and future decisions, and is really what the traditional scheduling problem is understood to be. The resulting implemented schedule based on the solution to several open-loop problems, is referred to as the closed-loop schedule or the closed-loop solution. The optimization model is kept identical for each open-loop problem, except for the updated parameter values. We 


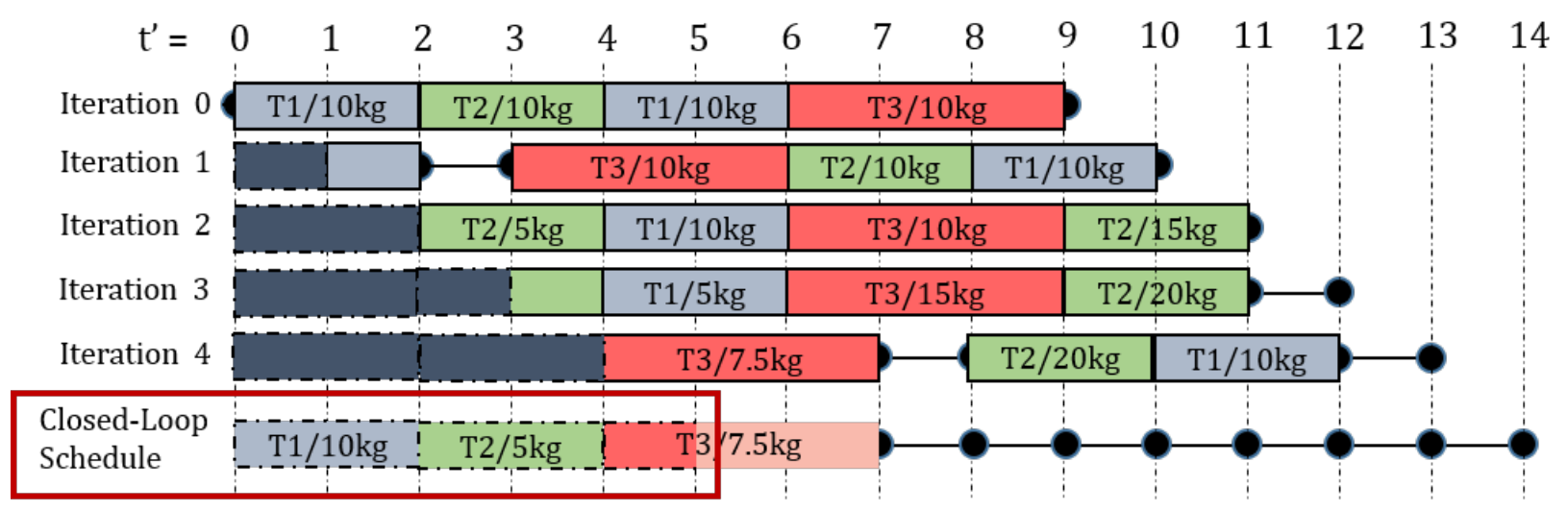

Figure 2: An example of closed-loop scheduling where a schedule is computed for next several time points but only the current decisions are implemented. The moving horizon is then moved forward and a new schedule is computed. Solid grey area denotes the region which is already implemented and hence fixed. Closed loop implemented schedule is shown in the bottom row.

define $t=0$, to always be the first time point of an open-loop optimization. We use an alias index $t^{\prime}$, to represent the time-grid for the open-loop optimization that just finished. An advantage of using the state-space model is that complete information about the current state of the system owing to current and past decisions is contained in variables at a single time point. Information about tasks that were underway, inventory and backlog at $t^{\prime}=1$ can be carried over as parameters to the next optimization's start time $t=0$ by fixing the corresponding variables (Fig. 3). To explain this concept, we label each open-loop optimization with an iteration number $\sigma \in\{0,1,2,3 \ldots\}$, and identify the variables in that optimization by writing the iteration number, as superscript on the left hand side. For example, variable $\bar{W}_{i j(t=0)}^{n}$ in iteration 23 , is written as ${ }^{(\sigma=23)} \bar{W}_{i j(t=0)}^{n}$. The variable values of optimizations that have been solved are stored as parameters. Equations 18a-18d show how variables for the current optimization $\sigma$ are fixed by equating them to the value of computed variables (now stored as parameters) from optimization $\sigma-1$. 


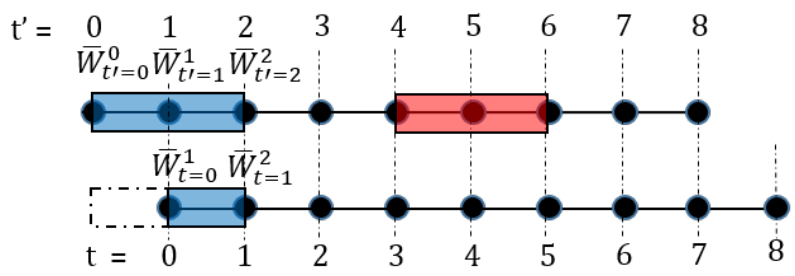

Figure 3: Carryover of lifted assignment $\left({ }^{(\sigma-1)} \bar{W}_{i j\left(t^{\prime}=1\right)}^{n}\right)$ information for a task under execution from previous optimization $\sigma-1$ as parameter to current optimization $\sigma$ to fix corresponding variable ${ }^{\sigma} \bar{W}_{i j(t=0)}^{n}$.

$$
\begin{aligned}
{ }^{\sigma} \bar{W}_{i j(t=0)}^{n} & ={ }^{(\sigma-1)} \bar{W}_{i j\left(t^{\prime}=1\right)}^{n} \quad \forall j, i \in \mathbf{I}_{j}, n \in\left\{1,2, \ldots . \tau_{i}\right\} \\
{ }^{\sigma} \bar{B}_{i j(t=0)}^{n} & ={ }^{(\sigma-1)} \bar{B}_{i j\left(t^{\prime}=1\right)}^{n} \quad \forall j, i \in \mathbf{I}_{j}, n \in\left\{1,2, \ldots \tau_{i}\right\} \\
{ }^{\sigma} S_{k(t=0)} & ={ }^{(\sigma-1)} S_{k\left(t^{\prime}=1\right)} \quad \forall k \\
{ }^{\sigma} B O_{k(t=0)} & ={ }^{(\sigma-1)} B O_{k\left(t^{\prime}=1\right)} \quad \forall k
\end{aligned}
$$

Note that ${ }^{\sigma} \bar{W}_{i j(t=0)}^{(n=0)}$ and ${ }^{\sigma} \bar{B}_{i j(t=0)}^{(n=0)}$, which represent degrees of freedom to start new tasks at $t=0$, are not fixed. Variables ${ }^{\sigma} \bar{W}_{i j(t=0)}^{n}$ and ${ }^{\sigma} \bar{B}_{i j(t=0)}^{n}$ are fixed for $n>0$ which represent lifted information of tasks that were already under execution. In addition, for the very first optimization, appropriate variables are also fixed to match the initial state of the plant. In this work, full rescheduling is carried out at every iteration, hence planned tasks from previous iteration, i.e. future decision variables (for times $t>1$ ) are not fixed.

If rescheduling is carried out at a rescheduling frequency $(\mathrm{RF})$ other than at every time point as described above, extending the aforementioned technique of fixing variables to reschedule every RF time points by advancing the moving horizon (consequently also by RF time points) is straightforward.

\section{Considerations in online scheduling}

In a dynamic environment with new incoming information, disruptive events or changes in resource availabilities, new schedules are computed and implemented. Although this practice 
of rescheduling is adopted routinely in production facilities, it is not known how it affects the overall performance of the production systems (Vieira et al., 2003). While formulating a lot-sizing formulation for reduced nervousness in production schedules Kazan et al. (2000) noted two factors that motivate rescheduling: the horizon effect and the forecast effect. The horizon effect is a deterministic effect that leads to rescheduling owing to the finiteness of the scheduling horizon. The forecast effect is a stochastic effect that leads to rescheduling owing to change in values of the stochastic parameters of the scheduling problem. As we show in the motivating examples to follow, even for the deterministic case it is not well understood how the characteristics of the open-loop problem affect the quality of the resulting closedloop schedule. Hence, studying the deterministic rescheduling problem even without any stochastic features is an important first step towards achieving better closed-loop scheduling.

\subsection{Motivating examples}

\subsubsection{Closed-loop vs. open-loop schedule}

To illustrate the difference between closed-loop and open-loop schedules, we consider process network-1 (Fig. 1) with orders due every 3 hours and order size of 4 tons of each product material (M5, M6, M7). The facility starts at $\mathrm{t}=0$ with a safety stock (inventory) of product materials sufficient to meet two orders. As discussed in Sec. 2.6, to compute a closed-loop schedule several open-loop problems need to be solved sequentially. We choose a moving horizon length $(\mathrm{MH})$ of 8 hours with cost minimization objective for the open-loop problem which is solved every hour. For the first open-loop problem, solved at $t=0$, there are only two orders within its horizon, one at $t=3$ hours and another at $t=6$ hours. Since the facility starts with inventory enough to meet two orders, no production is needed and hence the first open-loop problem has a completely empty schedule, with just the inventory holding costs of $\$ 26,400$ (Fig. 4A). The second open-loop problem however has its horizon spanning from $t=1$ to $t=9$ hours. Since the third order is due at $t=9$ hours, and there is inventory only to meet the first two orders, the second open-loop optimization yields a solution, wherein, production starts right away at $\mathrm{t}=1$ (Fig. 4B). Consequently, the closed-loop solution has no 
tasks starting at $\mathrm{t}=0$ but has a task starting at $\mathrm{t}=1$. The closed-loop solution resulting from solution to 8 open-loop problems (solved at $\mathrm{t}=0,1,2, \ldots 7$ respectively) is shown in Fig. $4 \mathrm{C}$. The cost of this closed-loop schedule is $\$ 27,393$. First and foremost, we note that the first open-loop problem solved spanning $t=0$ to $t=8$, and the closed-loop problem for the same time-span, have different schedules. Second, the costs of these schedules are different. This was however not very surprising, because the two problems saw different information. The first open-loop problem spanning $t=0$ to $t=8$ saw orders only for $t \leq 8$, while the closed-loop problem owing to the eight open-loop problem $(\mathrm{MH}=8)$ solved at $\mathrm{t}=7$, saw information up to $\mathrm{t}=15$. Thus we also find a solution to a single (long) open-loop problem spanning $\mathrm{t}=0$ to $\mathrm{t}=15$. The first 8 hours of this schedule is shown in Fig. 4D. If we evaluate the cost of this first 8 hours of schedule, it comes out to $\$ 29,592$. This schedule and its cost, still differ from the closed-loop schedule and the corresponding cost.

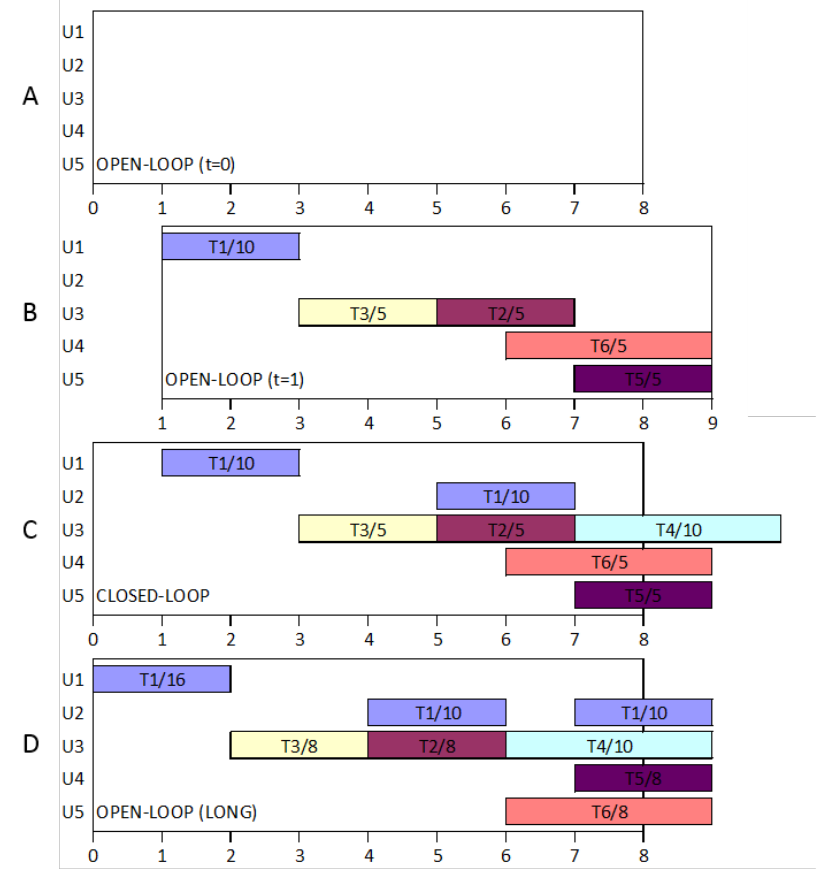

Figure 4: (A) Open-loop (at $\mathrm{t}=0$ ) with moving horizon length 8 hours, has an empty schedule and inventory holding cost of $\$ 26,400$. (B) Production starts in open-loop (at $t=1$ ) and the objective value for this openloop solution is $\$ 31,005$. (C) Resulting closed-loop schedule from solution to 8 open-loop problems (solved at $\mathrm{t}=0,1,2, \ldots 7$ respectively), with an evaluated cost of $\$ 27,393$. (D) Gantt chart for first 8 hours, of a long open-loop problem solved spanning $\mathrm{t}=0$ to $\mathrm{t}=15$.

In this example, it can be seen that the Gantt charts and cost values for closed-loop 
problem and open-loop problem are completely different. Therefore it is evident that the open-loop and closed-loop problems are different and knowing the solution to the former does not give the solution to the latter. The natural two questions then are: (1) How does the open loop optimization "determine" the closed-loop solution? (2) How can we modify the open-loop problem so that we obtain high-quality closed loop solutions?

\subsubsection{Empty closed-loop schedule}

Closed loop scheduling can present several paradoxes with regards to how the open-loop problem can affect the quality of the closed-loop schedule. To illustrate, we consider a very simple process network (Fig. 5) with one task, one unit, two materials and solve a profit maximization problem, wherein, sales are allowed at all times; with odd number of time-periods in the moving horizon, we can get an empty closed-loop schedule (Fig. 6A). This is because for the given network, with the task taking even number of time-periods for processing, but only odd number of time-periods being available in the scheduling horizon, multiple optimal solutions exist, and one of them is to have two tasks start after a single idle time-period. This open-loop solution, leads to an empty closed-loop schedule, because task start decisions are always postponed and never implemented.

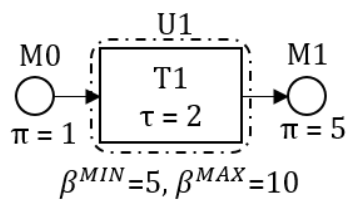

Figure 5: Network with one task, one unit and two materials (Process Network-2: PN2).

To circumvent this problem, an ad-hoc fix that can be applied to the open-loop problem is to left-shift open-loop schedules. This can be achieved by tailoring the open-loop objective to favor early sales over sales towards the latter part of the moving horizon (greedy objective). Thus we get a closed-loop schedule, which is not empty (Fig. 6B). Although, here through an objective function modification we could fix the problem, in the general case it is not known what modifications will be needed to obtain a good closed-loop solution. 


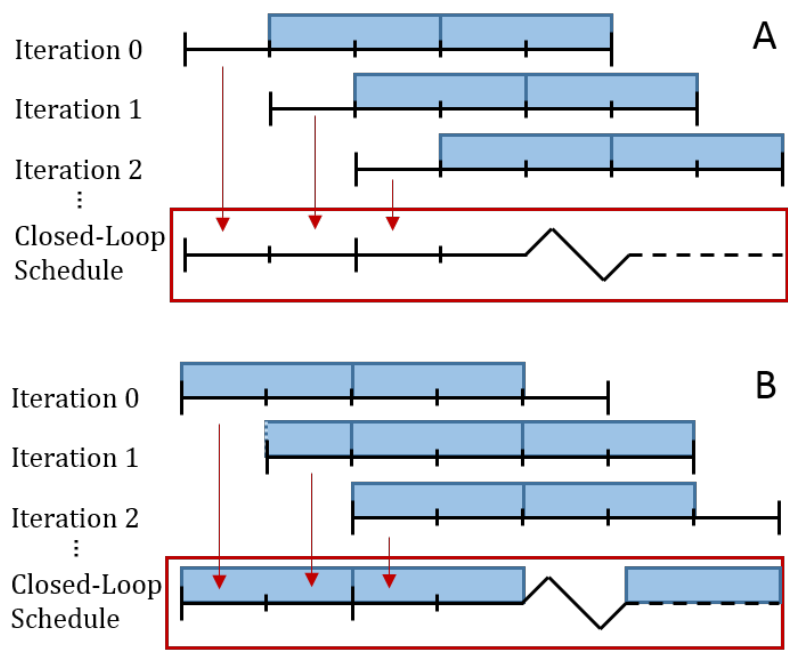

Figure 6: (A) Moving horizon with odd number of time-periods for PN-2 (Fig. 5) gives an empty closedloop schedule. (B) Left shifting of tasks by making open-loop objective greedy gives a non-empty closed-loop schedule.

\subsubsection{Optimality of open-loop}

Let us consider process network-3 (Fig. 7) with orders of size 3 tons for each product material due every $3 \pm 1$ hours (integer uniform distribution; refer to Sec. 4.1.2 for detailed explanation of how demand patterns are generated). The objective is to maximize profit. Excess-sales are allowed only at those times when orders are due.

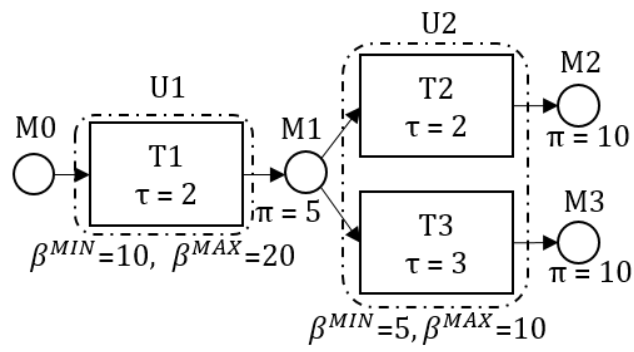

Figure 7: Network with two units, three tasks, and four materials (Process Network-3: PN3). Task T1 can be carried out in unit 1 while task T2 and T3 compete for being processed on unit 2 .

The open-loop objective is modified such that early sales bring more profit than latter sales so that tasks are left-shifted, and excess product inventory is shipped out as soon as possible. If excess-sales are not committed early on in the moving horizon, excess production accumulates as inventory. The favoring of early sales in the open-loop objective however is an artificial tweak to guide the optimizations to produce and sell early; therefore while 
subsequently measuring closed-loop quality all sales are counted towards bringing same profit per unit of material sold.

Since task 2 takes less time to execute than task 3, material 2 can be produced at a faster rate. Furthermore, since products sell at the same price, executing task 2 leads to more profit than task 3 , so every time task 3 is executed more than the minimum needed, 1 hour of time is "lost". Hence, the best schedule is where task 2 dominates the schedule and task 3 has the minimum possible number of executions, just sufficient to meet the demand for material 3.

A closed-loop schedule for 1 week obtained with scheduling horizon (moving horizon length) of 12 hours, and with each open-loop optimization solved to optimality (OPTCR=0\%), has 25 executions of task T3. Surprisingly, when each open-loop optimization is solved with OPTCR $=5 \%$, the number of executions for T3 in the closed-loop schedule reduces to 21 ! In other words, a suboptimal solution to the open-loop problem can actually lead to a better closed-loop solution! The two Gantt charts showing the closed-loop schedule for 1 week for unit 2 are shown in Fig. 8. Figure 9 shows the cumulative sum of shipments of material 3 for the two closed-loop schedules (optimal and suboptimal) and the minimum required as per the approximately periodic demand.

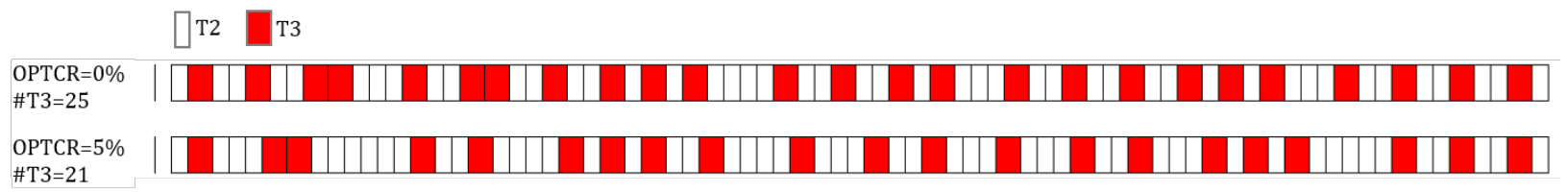

Figure 8: Closed loop schedule for unit 2 obtained using optimality gaps $0 \%$ and $5 \%$; with OPTCR $=0 \%$, $\mathrm{T} 3$ is executed 25 times, while, with $\mathrm{OPTCR}=5 \%$, T3 is executed 21 times. Less number of executions of T3 is better.

As we will discuss subsequently in Sec. 7.3, this unexpected improvement in closedloop solution quality can be explained and addressed. However, as problems scale up in complexity, several paradoxes can be concurrently present which can lead to very inefficient closed-loop schedules. Hence, it is important to study and better understand the factors that affect the quality of closed-loop schedules. 


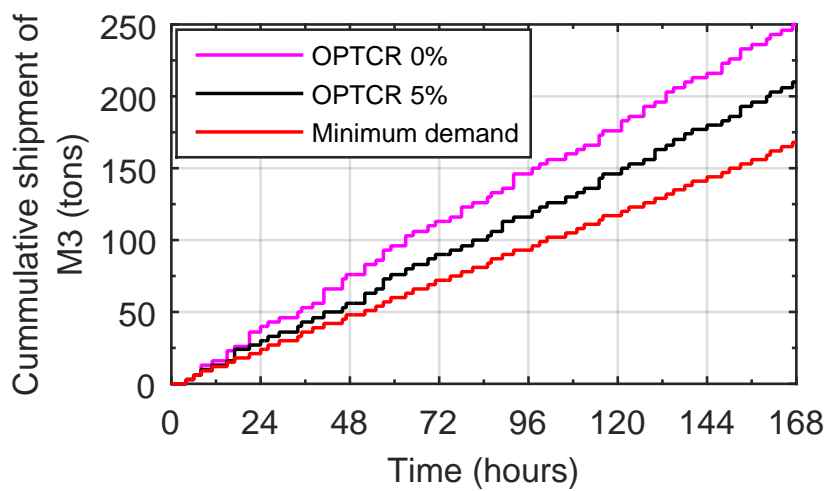

Figure 9: Less favorable material M3 is produced more in closed-loop schedules when OPTCR=0\% (magenta). Black represents OPTCR $=5 \%$. Production and shipment closer to minimum needed (red) is better.

\subsection{Online scheduling design attributes}

\subsubsection{Rescheduling frequency}

Rescheduling is traditionally seen as an event-triggered activity wherein an event could be the arrival of an order, a unit breakdown, processing delays, etc. However, we reason that rescheduling should also be performed due to the addition of time (i.e., resource availability change) and not just when orders change, units breakdown or when tasks are delayed. However, rescheduling has an implicit cost associated with it due to plant nervousness that it induces (Kopanos et al., 2008; Novas and Henning, 2010). Hence it is important to quantify how online scheduling affects the quality of the implemented closed-loop schedule, and then decide how often rescheduling be performed.

\subsubsection{Moving horizon length}

In addition to rescheduling frequency, a key characteristic of the open-loop problem is the scheduling horizon length because it dictates if orders farther into the future are accounted for while computing the current schedule. A myopic horizon can lead to bad early decisions, necessitating costly revised schedules in the future. In general, it might be a good idea to account for as long a scheduling horizon as possible to have full information availability about future orders. In practice however, longer horizons increase the problem size and might render computing good schedule challenging in real-time. Hence it is natural to ask 
what should be an appropriate length for the moving horizon and on what problem instance features does it depend on.

\subsubsection{Suboptimal solution to online optimizations}

Solving large scale scheduling problems can be computationally very expensive. Due to the limited time available for online computations, it might not be possible to solve the models to optimality. In general, it has been assumed that the closed-loop solution would deteriorate due to accumulation of these suboptimalities. However, there is no study showing that this is indeed the case. In fact, it could also be argued that suboptimal moves will be corrected due to feedback, so the closed-loop schedule will remain minimally affected. Furthermore, due to the finite moving horizon of the open-loop problem, it cannot be directly deduced that the open-loop optimization should even be attempted to be optimally solved, especially since suboptimal solutions can be obtained faster allowing us to reschedule more

frequently. Accordingly, one of the specific aims of this paper is to study the effect of suboptimal open-loop optimizations on the quality of the resulting closed-loop schedule.

\subsubsection{Other considerations}

In addition to rescheduling frequency, moving horizon length and suboptimal solution of open-loop problem, there are additional approaches, such as open-loop objective function modifications and possible added constraints that can be adopted to improve closed-loop solution quality. In the motivating examples, we showed that modifications in the objective function can lead to improvements or deterioration in closed-loop quality. It remains to investigate if certain constraints can possibly be added to the open-loop problem to get improved closed-loop solutions.

\subsection{Key questions}

To summarize, some intriguing questions the answers to which can lead to better understanding of closed-loop scheduling are as follows:

(i) Should online scheduling be carried out periodically as a time-driven activity? 
(ii) How frequently should schedules be revised? Does the frequency depend on instance characteristics such as the frequency at which orders are due?

(iii) What is an appropriate length of moving horizon and on what instance characteristics does it depend on?

(iv) How does suboptimalilty in each open-loop optimization problem affects the quality of closed-loop solution?

(v) Could the open-loop problem be modified in terms of its objective and constraints to give better closed-loop schedules?

\section{Simulations}

\subsection{Problems, instance types, instances and runs}

Once problem features are fixed (variable batch-sizes, utilities, setups), a problem is defined by the objective function (OBJ). An instance type, is a problem expressed for a process network (PN) and a demand pattern (D). A demand pattern, which is further discussed in Sec. 4.1.2, is a distribution of order due-times and sizes of each order. For each instance type (OBJ.PN.D), we define an instance designated by a sample (S) from the demand pattern under consideration. Finding the closed-loop solution for a given instance, using a design attribute tuple (RF.MH.OPTCR), where RF is rescheduling frequency, $\mathrm{MH}$ is moving horizon length, and OPTCR is the relative estimated gap at which the branch and bound solution algorithm is terminated, is referred to as a problem run, or simply a run. A run can be represented as (OBJ.PN.D).(S).(RF.MH.OPTCR). Each run requires solution to several open-loop problems solved sequentially to find the closed-loop solution.

\subsubsection{Objective functions and process networks}

We consider PN-3 showed in Fig. 7 with cost minimization and profit maximization as objective functions (Eqs. 14a, 14b). We also validate our findings using PN-1 (Fig. 1), and 
present these as extended results in supplementary information. For profit maximization we enforce an additional constraint, to allow excess-sales of product materials only at those times when orders are also due $\left(\xi_{k t}=0 \Longrightarrow V_{k t}^{S}=0\right)$.

\subsubsection{Short term demand patterns}

We generate short-term demand patterns using four attributes: (i) Expected time-period between order due-times, denoted by $p$; (ii) Variation of order due-times around the expected due-times, denoted by $\Delta p$; (iii) Expected demand of each product in an order, denoted by $m$; (iv) Variation in order size around the expected size, denoted by $\Delta m$. Hence, a 4-tuple $(p, \Delta p, m, \Delta m)$ characterizes a demand pattern. Samples from these patterns combined with a process network and scheduling objective define problem instances. Figure 10(A) shows a demand pattern and Fig. 10(B) shows one sample of demand from this pattern.

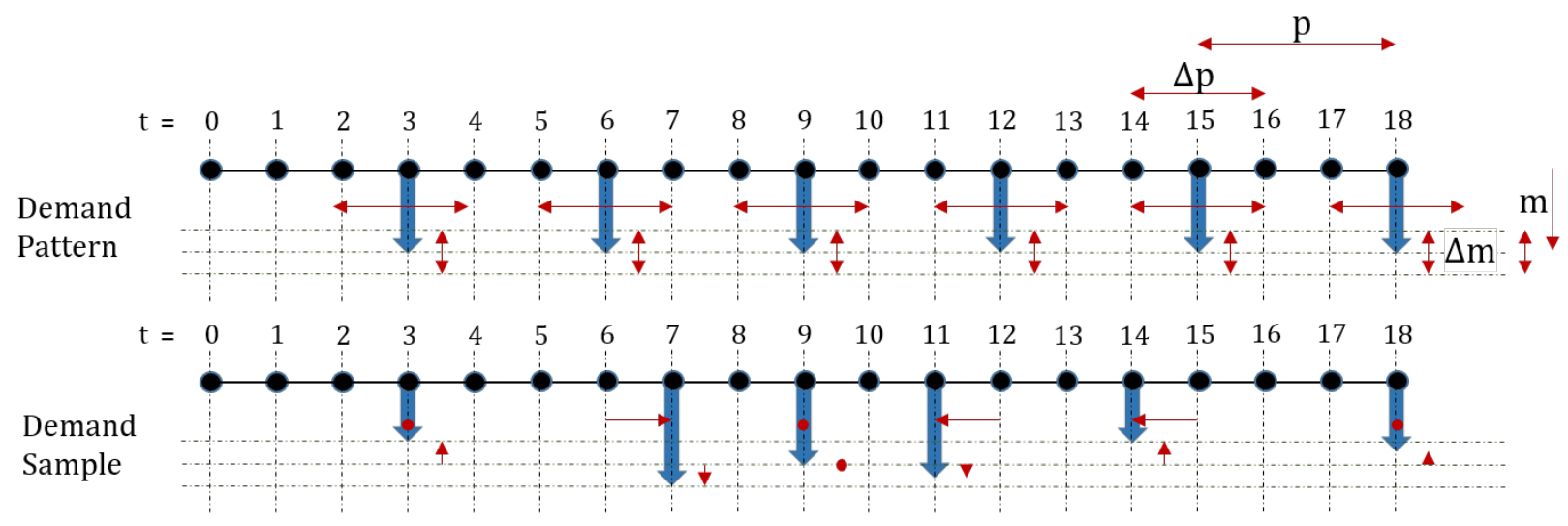

Figure 10: (A) Short term demand patterns are generated by first assigning orders $l \in \mathbf{L}$ due-times on regular periodic time intervals $t_{l+1}=t_{l}+p$ with demand of $m$ tons of each product in every order. (B) Each order's due-time is then sampled from a discrete uniform distribution $\left[t_{l}-\Delta p, t_{l}+\Delta p\right]$, and demand for each product in that order, sampled from a continuous uniform distribution $[m-\Delta m, m+\Delta m]$. This creates the size and due-time of the orders irregular, which is a characteristic of short-term scheduling. In the figure, $p=3$ hours, $m=1$ ton, $\Delta p=1$ hour, and $\Delta m=0.25$ ton. Arrows with head on both ends represent span of distributions. Arrows with head on one end, represent the deviation from mean for the sample, while the small disks represent zero deviation from mean for the sample.

A short term demand pattern, generates an average $m / p$ tons per hour of production load of each product. For PN-3, for cost minimization, we choose three values of $p=$ (3), (6), (12), and the corresponding $\Delta p=(1),(1),(3)$. For each of these $p$, we choose three $m$ values, such that the network facility is on an average loaded to approximately $50 \%$, 
$75 \%$, and almost $100 \%$ of its production capacity. Thus, for each $p=(3),(6),(12)$, we choose $m=(3,4,5),(6,8,10),(12,16,20)$, and for each $m$, the corresponding $\Delta m=0.1 m$, i.e. $10 \%$ variation in order sizes. For profit maximization, shipments through excess-sales over and above minimum demands are allowed, hence the facility is expected to be running at full production capacity throughout. Thus for each $p=(3),(6),(12)$, we choose only one $m=(3),(6),(12)$, such that if only this minimum demand was to be met, the network facility would be running at approximately $50 \%$ of its capacity. We choose the corresponding $\Delta p=(1),(1),(3)$ and $\Delta m=0.1 m$.

\subsubsection{Design attributes}

We investigate the quality of closed-loop schedules as a function of rescheduling frequency $(\mathrm{RF})$, moving horizon length $(\mathrm{MH})$, and relative estimated optimality gap (OPTCR) of each optimization, by varying one of these design attributes at a time while keeping the others fixed. We consider $\mathrm{RF}=1,2,3,4,5,6$ hours; $\mathrm{MH}=18,21,24,27,30,33,36$ hours; and $\mathrm{OPTCR}=0 \%, 2.5 \%, 5 \%, 7.5 \%, 10 \%$.

\subsection{Scheduling model and parameters}

The state-space model described in Sec. 2.4 is used for computing closed-loop schedules. Each network facility starts with inventory to meet nominal demand for the first 12 hours $\left(S_{k(t=0)}=12 \frac{m}{p}\right)$. A discrete time-grid with spacing of $\delta=1$ hour is used. Each task has a fixed production cost $\left(\alpha_{i j}^{P}=\$ 1\right)$ but no proportional cost $\left(\alpha_{i j}^{P}=0\right)$. Inventory $\operatorname{costs}\left(\pi_{k}^{I N V}\right)$

are 10 times that of backlog $\operatorname{costs}\left(\pi_{k}^{B O}\right)$, and both are directly proportional to material price $\left(\pi_{k}\right)$

From every open-loop computation, only the first RF scheduling decisions are implemented. The moving horizon is then advanced forward by RF time points and a new schedule is computed. Through this procedure, closed-loop schedules are obtained for seven days (168 hours). Hence, a total of $\lfloor 168 / \mathrm{RF}\rfloor$ number of optimizations/computations are carried out to obtain a single closed-loop schedule. 


\subsection{Scaling and analysis}

The closed-loop solution from each run (OBJ.PN.D).(S).(RF.MH.OPTCR) yields a closedloop objective. For a given instance type (OBJ.PN.D), we sample $n$ demands $\left(\mathrm{S}_{1}, \mathrm{~S}_{2} \ldots \mathrm{S}_{n}\right)$ to generate $n$ instances. For each instance, we solve several runs over the space of design attributes (RF.MH.OPTCR) to get corresponding closed-loop objective values.

We then take the mean of the closed-loop objective values obtained from these runs over demand samples $\left(\mathrm{S}_{1}, \mathrm{~S}_{2}, \ldots \mathrm{S}_{n}\right)$, and scale them within the given instance type (OBJ.PN.D), by the mean closed-loop objective value corresponding to the tuple with the longest moving horizon, the fastest rescheduling frequency and the smallest OPTCR, since this tuple is expected to yield the best closed-loop solution. The scaled closed-loop objective values now show a qualitative trend of how the mean closed-loop solution improves or deteriorates for different design attribute tuples for the chosen instance type.

Within an instance type (OBJ.PN.D) we use the same set of $n$ demand samples for finding closed-loop solutions for all design attributes, rather than sampling a different set

of $n$ demand samples. This (matched) dependent sampling leads to stronger statistical inferences in less number of samples than if the samples were to be (unmatched) independent (Wonnacott and Wonnacott, 1972). For our investigation, we choose number of demand samples, $n=10$.

\subsection{Computing resources}

Approximately 20,000 closed-loop schedules were obtained for this study. To obtain each closed-loop schedule (a single run), the requisite number of optimizations are carried out sequentially using default solver options in CPLEX 12.6.1 via GAMS 24.4.3. A set of 24 cluster machines with Intel Xeon $(\mathrm{E} 5520,2.27 \mathrm{GHz}, 8$ core) processors, 16 GB of RAM and Linux CentOS 7 operating system were used in parallel. HTCondor software (HTCondor 8.5.0, 2015) was used for queuing and managing all computation jobs on these cluster machines. GDXMRW data exchange library (Dirkse et al., 2014) was used to import, output data from GAMS gdx data files, for analysis in MATLAB R2015a. 


\section{Results}

In Figs. 11-16, each subplot corresponds to a single instance type, and carries a tag of the form $\mathrm{F} p \mathrm{~L} m$ which denotes that orders are due, on an average every $p$ hours, and each order has an average size of $m$ tons for each product. For example, F6L8 means orders are due, on an average, every 6 hours with mean order size of 8 tons of each product. Table 1 shows the values of $\mathrm{F} p \mathrm{~L} m$ used and the corresponding approximate production capacity utilization for PN-3 (Fig. 7). The frequency at which orders are due increases from F12 to F6 to F3. This

Table 1: Values of FpLm and their corresponding production capacity utilization for PN-3 (Fig. 7).

\begin{tabular}{lcc}
\hline F $p$ L $m$ & Normalized demand & Load relative to capacity (approx.) \\
\hline F3L3, F6L6, F12L12 & 1.00 ton/hr & $50 \%$ \\
F3L4, F6L8, F12L16 & 1.33 ton/hr & $75 \%$ \\
F3L5, F6L10, F12L20 & 1.67 ton/hr & $100 \%$ \\
\hline
\end{tabular}

is because the convention followed for describing frequency $x$, is orders due every $x$ hours as opposed to $1 / x$ hours $^{-1}$. The scaling in each subplot is with respect to the data within that subplot, and hence scaled quality numbers cannot be compared across subplots. For example, a quality of 1 in a given subplot is different in absolute objective value than quality of 1 in any other subplot. In addition, making a direct comparison between the results from different subplots is unjustifiable because they are subject to a different demand pattern (D).

We carry out a two-way analysis of variance (two-way ANOVA) for our results in Figs.

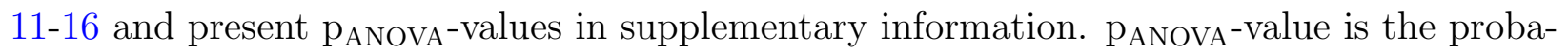
bility of realization of observed data if the null hypothesis was true. We formulate our null hypothesis to be that the design attribute under consideration has no effect on closed-loop quality. Where an effect of design attribute is observed, pANOvA-values are less than $5 \%$ indicating a statistically discernible effect. In addition, we also find Spearman correlation

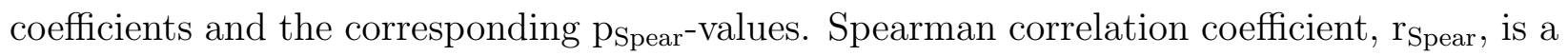
metric of monotonic dependence of observed data on the attribute being varied. For example, if closed-loop cost strictly decreases with longer moving horizon length, we get a Spearman 


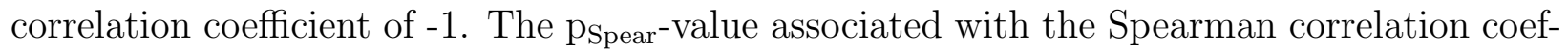
ficient indicates, how well the increase/decrease in observed data is explained by regression. Where an increase/decrease is explained well by regression, the corresponding p $_{\text {Spear-values }}$ are less than $10 \%$.

\subsection{Rescheduling frequency}

\subsubsection{Cost}

Figure 11 shows how closed-loop quality for cost minimization is affected by rescheduling frequency. Each open-loop problem is solved to optimality. The overall trend is that when rescheduling is carried out faster the closed-loop quality improves (cost reduces). This observation holds irrespective of the frequency at which orders are due, thus suggesting that rescheduling should be carried out also as a time-driven activity to account for the advancing horizon, rather than just an event-triggered activity. For longer moving horizon lengths $(\mathrm{MH}=24,30)$ the closed-loop quality is minimally affected by rescheduling frequency. This is because the moving horizon is long enough to account for orders in the future, for which production needs to be scheduled now.

\subsubsection{Profit}

Figure 12 shows how the closed-loop quality for profit maximization is affected by rescheduling frequency. It can be seen that faster rescheduling yields higher closed-loop profit for each demand pattern. This is surprising because, for the facility running anyways at its $100 \%$ capacity, we would expect that the arrival of new orders would not have an effect on optimality of the incumbent schedule. This however is not true. Decisions to sell extra inventory need revisions with the advancement of the moving horizon. Hence, faster rescheduling provides better inventory management through revision of excess-sales decisions which were made originally without the knowledge of incoming orders. This also explains why for a longer moving horizon length (here $\mathrm{MH}=30$ ), rescheduling faster does not make much difference because the excess-sales decisions are already far-sighted. 


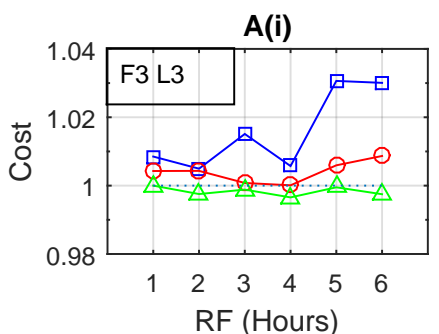

A(ii)

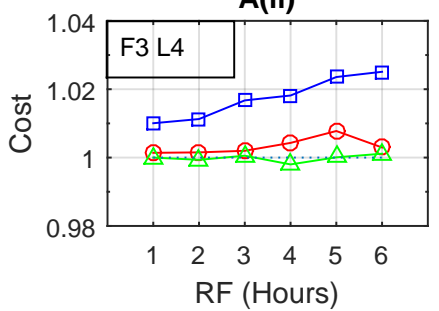

A(iii)

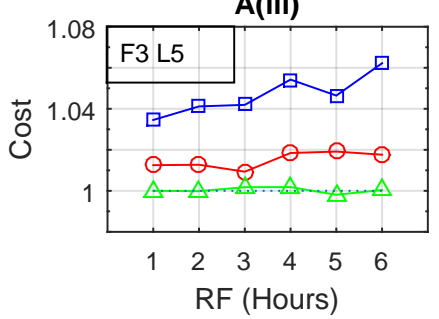

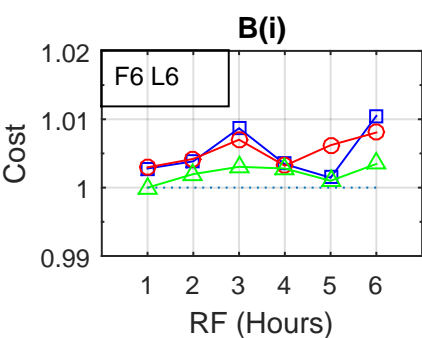

B(ii)

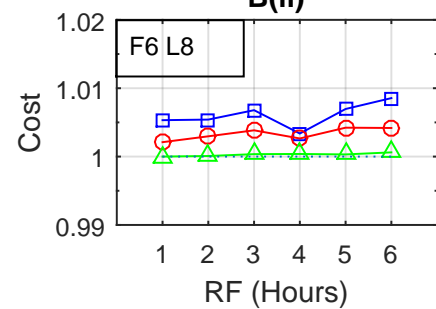

B(iii)

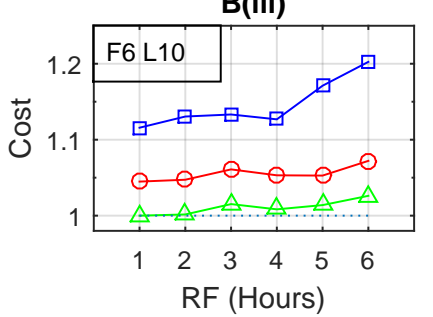

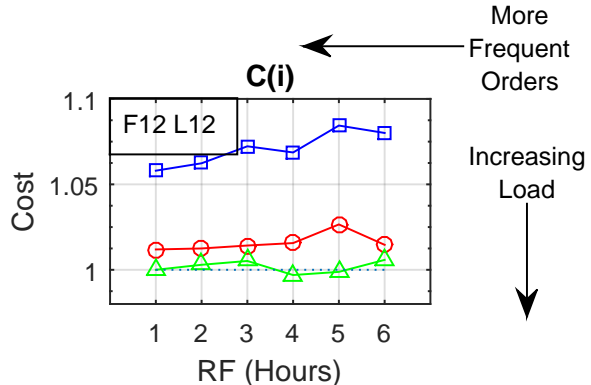

C(ii)

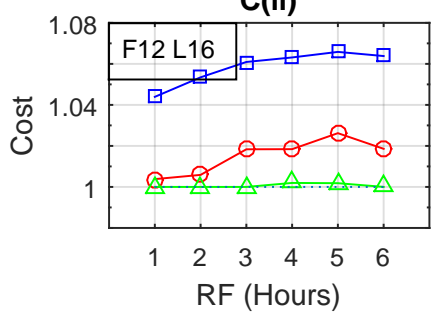

C(iii)

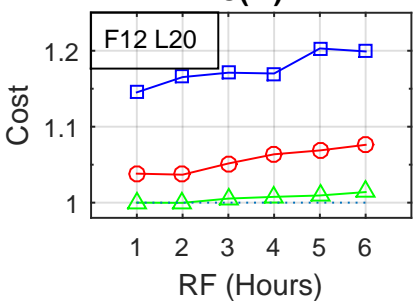

- MH $18-0-$ MH $24-\triangle$ MH 30

Figure 11: Effect of rescheduling frequency for cost minimization objective. Each data point is an average cost of closed-loop schedules for 10 demand samples, and is scaled by mean closed-loop cost corresponding to $\mathrm{MH}=30, \mathrm{RF}=1$ within each subplot.
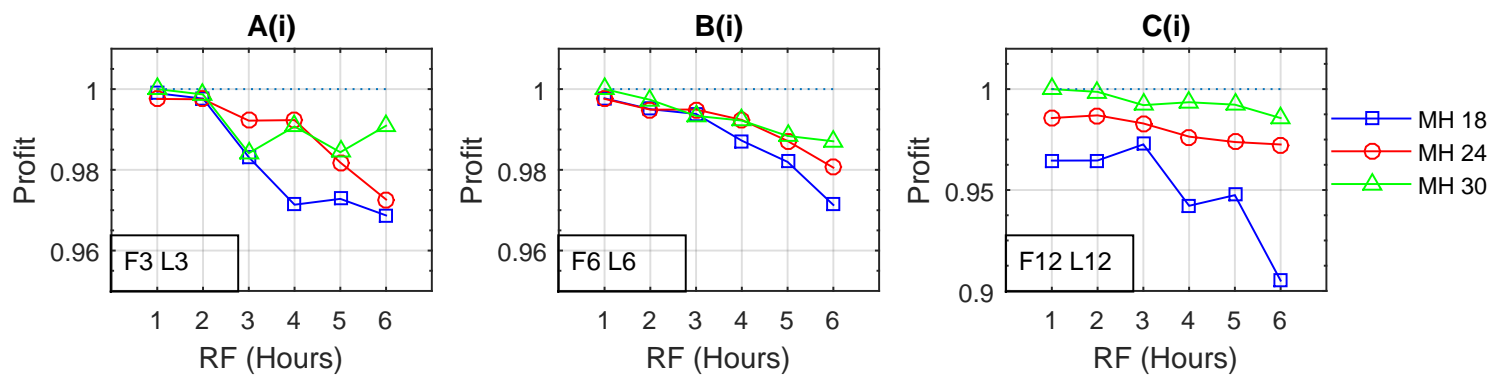

Figure 12: Effect of rescheduling frequency for profit maximization objective. Each data point is an average profit of closed-loop schedules for 10 demand samples, and is scaled by mean closed-loop profit corresponding to $\mathrm{MH}=30, \mathrm{RF}=1$ within each subplot. 


\subsection{Moving horizon length}

\subsubsection{Cost}

Figure 13 shows how the closed-loop quality for cost minimization is affected by moving horizon length. Each optimization problem is solved to optimality. The overall trend is that as the moving horizon length increases the closed-loop quality improves. Interestingly, for $100 \%$ loads, a shorter moving horizon leads to more deterioration in quality than when the load is $50 \%$ or $75 \%$ of the production capacity. To meet a big order, multiple batch executions are needed. When these big orders are temporally spaced out (infrequent), a short moving horizon, owing to its limited ability to look ahead, is unable to start production in a timely manner to meet these orders, thus we see a large deterioration in closed-loop quality. Figures 13B(i),B(ii) show almost identical closed-loop quality for all moving horizon lengths.

\subsubsection{Profit}

Figure 14 shows how the closed-loop quality for profit maximization is affected by moving horizon length. It can be seen that longer moving horizon lengths do not necessarily lead to substantially higher closed-loop profit, unless orders are due infrequently (Fig. 14C(i)), or rescheduling is slow. Since the facility is pushed to operate at $100 \%$ capacity anyways, knowledge of future orders does not influence the current production schedule in a significant manner, other than revision of excess-sales decisions. Thus, as expected, to have taken better inventory management decisions (excess-sales), a slower rescheduling frequency (here $\mathrm{RF}=6$ ) benefits more from longer moving horizon lengths than a faster rescheduling frequency $(\mathrm{RF}=1)$. 


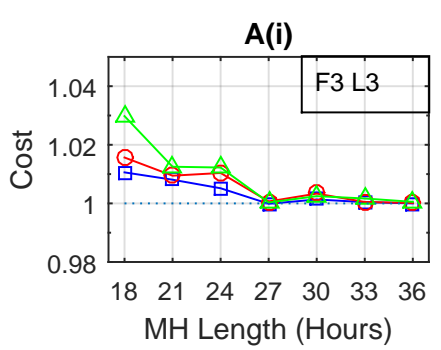

A(ii)

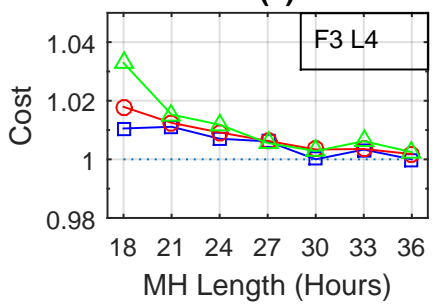

A(iii)

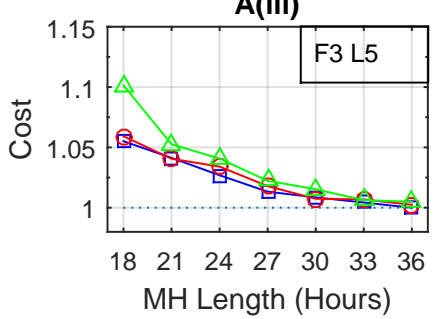

$\mathrm{B}(\mathrm{i})$

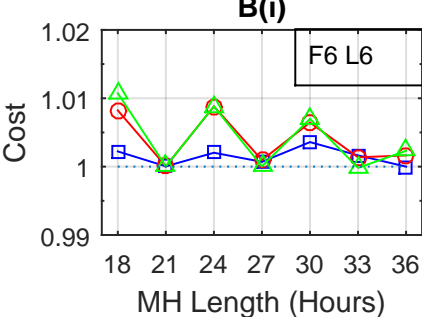

B(ii)

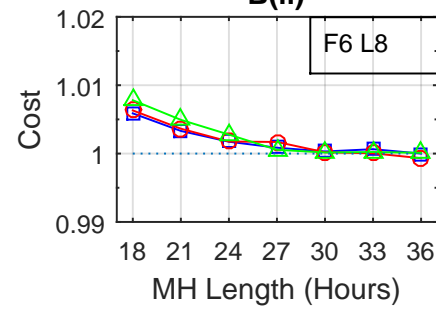

B(iii)

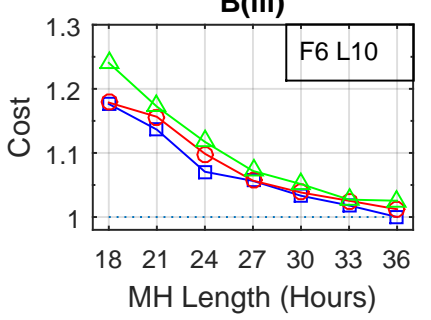

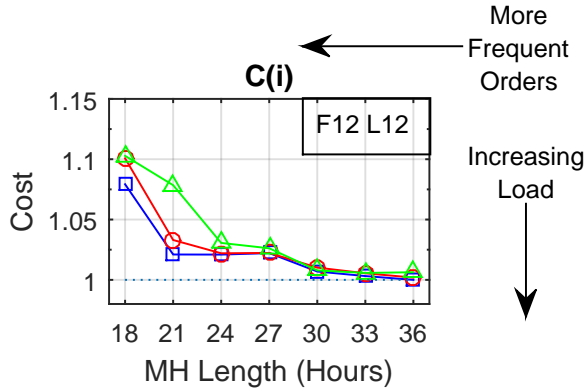

C(ii)

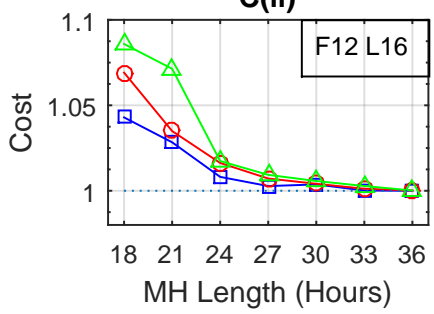

C(iii)

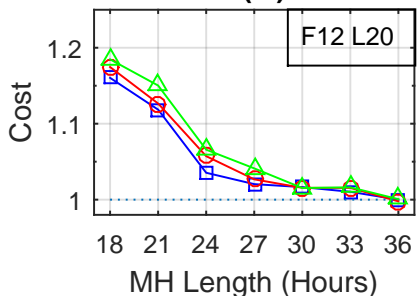

曰-RF $1-$ RF $3-\triangle$ RF 6

Figure 13: Effect of moving horizon length for cost minimization objective. Each data point is an average cost of closed-loop schedules for 10 demand samples, and is scaled by mean closed-loop cost corresponding to $\mathrm{MH}=36, \mathrm{RF}=1$ within each subplot.
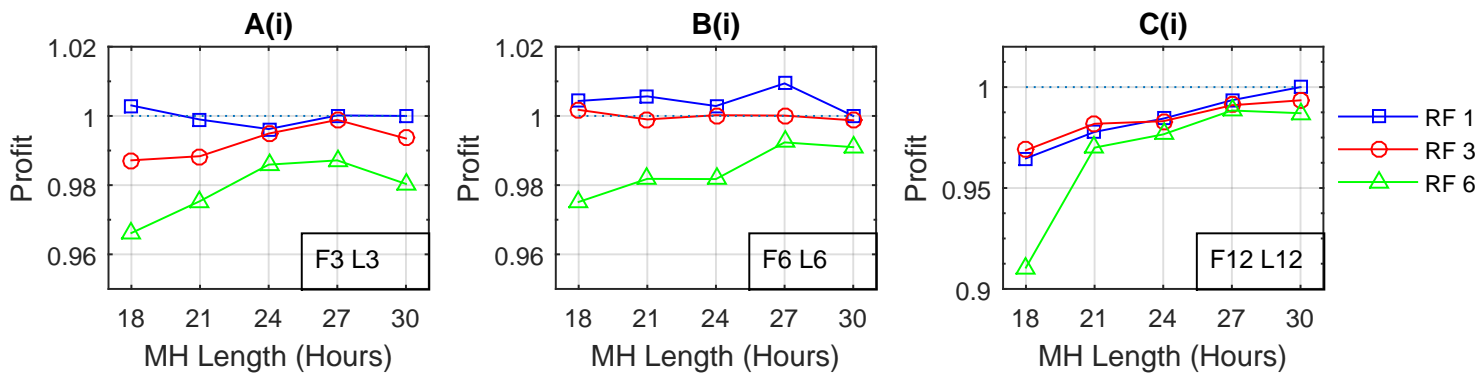

Figure 14: Effect of moving horizon length for profit maximization objective. Each data point is an average profit of closed-loop schedules for 10 demand samples, and is scaled by mean closed-loop profit corresponding to $\mathrm{MH}=30, \mathrm{RF}=1$ within each subplot. 


\subsection{Suboptimality}

\subsubsection{Cost}

Figure 15 shows how the closed-loop quality for cost minimization changes with the relative optimality gap (OPTCR). We present results with $\mathrm{MH}=24$ hours, but $\mathrm{MH}=12$, 18, and 30 hours also exhibit qualitatively identical trends. The overall trend is that as OPTCR increases, closed-loop quality deteriorates, but only slightly. Specifically, the percentage deterioration in closed-loop quality is much less than the corresponding percentage of OPTCR. For example, in Fig. 15B(ii) we see about 1\% deterioration in closed-loop quality for $\mathrm{OPTCR}=10 \%$ and negligible deterioration for OPTCR $=5 \%$. An interesting observation is that frequency and size of orders, do not appear to influence the magnitude of deterioration due to suboptimal computations.
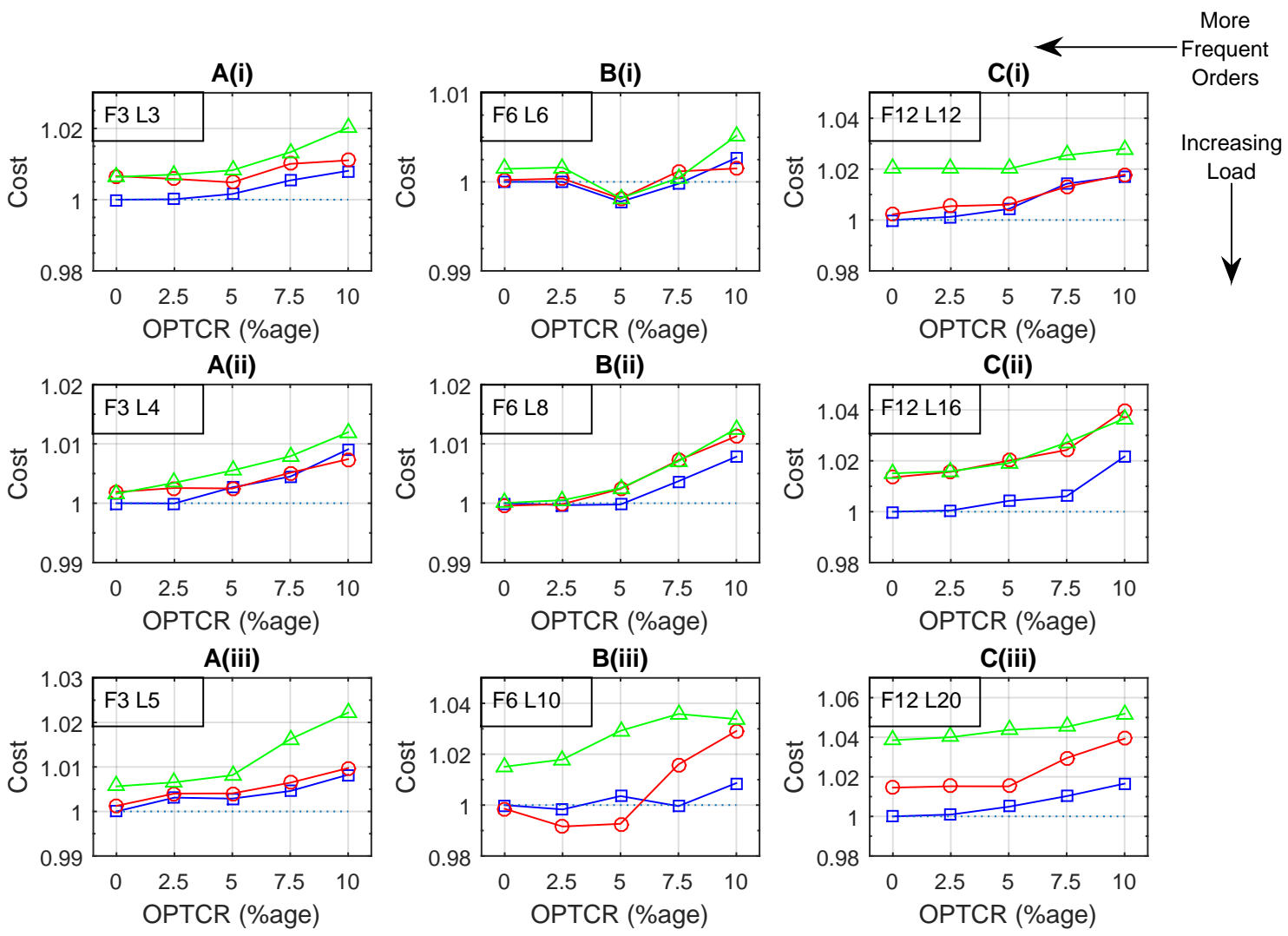

$\square$ - RF $1-0-$ RF $3-\triangle \mathrm{RF} 6$

Figure 15: Effect of OPTCR for cost minimization objective. Moving horizon length of 24 hours is used. Each data point is an average cost of closed-loop schedules for 10 demand samples, and is scaled by mean closed-loop cost corresponding to $\mathrm{OPTCR}=0 \%, \mathrm{RF}=1$ within each subplot. 


\subsubsection{Profit}

Profit maximization (Fig. 16) shows similar results as cost minimization: closed-loop profit deteriorates as OPTCR increases. However, the deterioration in closed-loop quality is much less than the OPTCR. For example, in Fig. $16 \mathrm{~B}(\mathrm{i})$ for $\mathrm{RF}=1$ we see less than $1 \%$ deterioration in closed-loop profit for OPTCR=10\%. Even for slower rescheduling frequencies $(\mathrm{RF}=3$ or 6$)$ we see less than $4 \%$ deterioration in profit for OPTCR $=10 \%$. For OPTCR $=5 \%$ there is an insignificant deterioration.
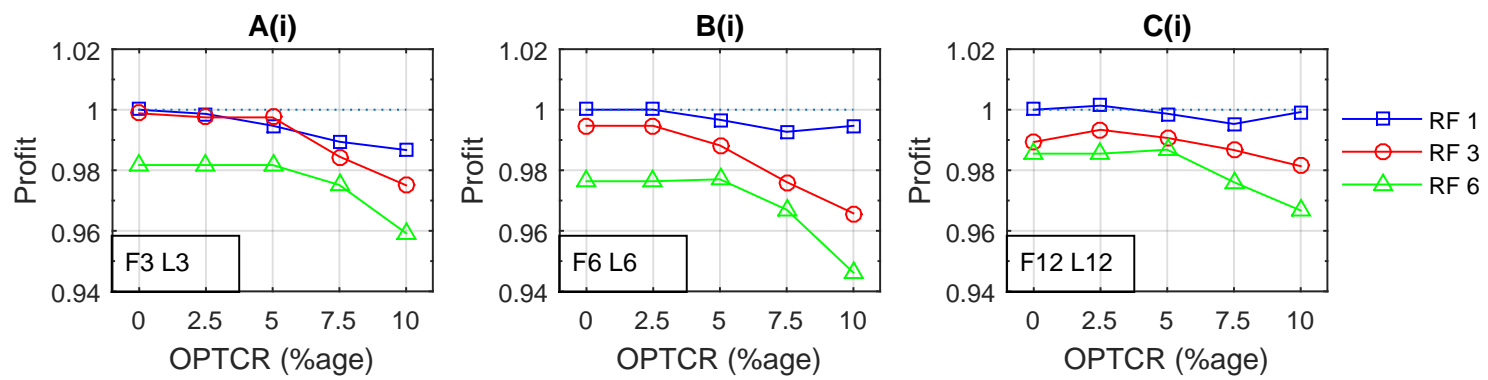

Figure 16: Effect of OPTCR for profit maximization objective. Moving horizon length of 24 hours is used. Each data point is an average profit of closed-loop schedules for 10 demand samples. Moving horizon length of 24 hours is used, and is scaled by mean closed-loop profit corresponding to OPTCR $=0 \%, \mathrm{RF}=1$ within each subplot.

\subsubsection{Estimated vs. true gap}

To understand these disproportionately small deteriorations in closed-loop quality due to non-zero OPTCR, we present in Fig. 17, the mean relative estimated gaps (Eq. 15) and mean relative true gaps (Eq. 16), over all the open-loop optimizations carried out to produce results in Figs. 15 and 16. We see that true gaps are surprisingly much smaller than the relative estimated gaps. For example, for cost minimization, we see that for OPTCR $=5 \%$, the true gaps in the open-loop optimizations are on an average $0.24 \%$, and the resulting average deterioration in closed-loop quality is $0.22 \%$. 

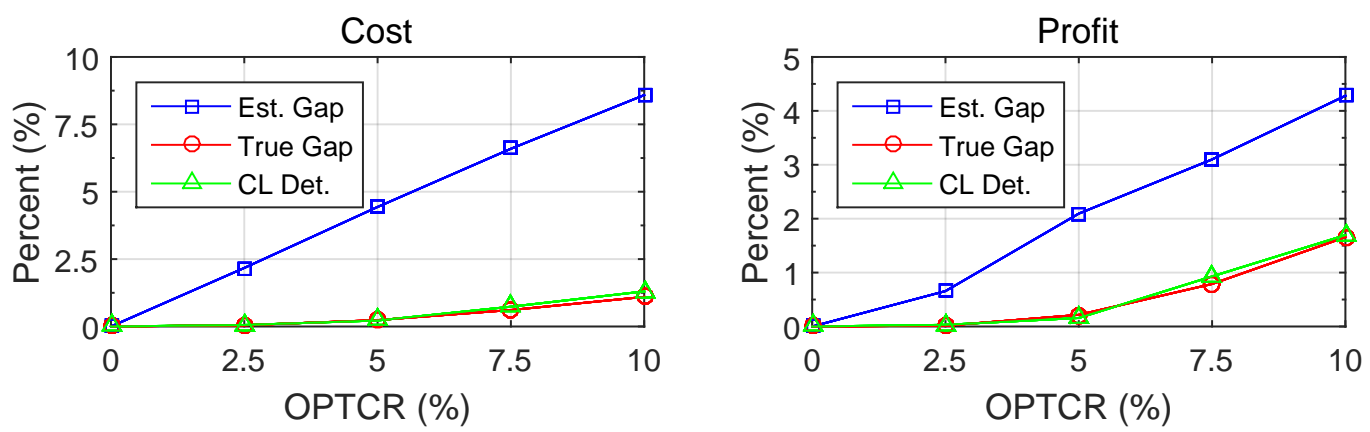

Figure 17: Mean estimated relative gap (Est. Gap) and mean true relative gap of open-loop optimizations (True Gap), and the resulting mean closed-loop deterioration (CL Det.), as a function of OPTCR. Mean integrality gap (Eq. 17) for cost minimization is $45.4 \%$, and for profit maximization is $3.7 \%$.

\section{Discussion}

\subsection{Rescheduling frequency}

In general, higher rescheduling frequency leads to better closed-loop quality, however there exists an upper threshold, beyond which closed-loop performance does not improve. Importantly, lower than a certain frequency leads to very bad closed-loop schedules. One of the factors on which this lower threshold depends, is the frequency of orders. If orders are due frequently, then rescheduling infrequently leads to poor closed-loop schedules. Even when orders are due infrequently, it is still important to reschedule periodically. Hence for the lower threshold what matters is both, the frequency at which orders are due, and the length of time by which the moving horizon advances.

\subsection{Moving horizon length}

An appropriate length for the moving horizon depends on both, the frequency of orders and the time needed to meet an order. Short moving horizons could be myopic, thus unable to take good current decisions. Also, lengths beyond a certain threshold do not result in any improvement, because in this case, orders located in the latter part of the long moving horizon are already much further out thus not affecting decisions that will be immediately implemented. The lead time to meet a new order is itself a function of the order size and the current load on the shared facility. A heavily loaded facility would have longer lead 
times, while a lightly loaded facility would have underutilized capacity, possibly allowing fast response to meet new orders with short lead times.

\subsection{Suboptimal solution to open-loop problem}

An OPTCR lesser than a certain threshold (in our results 5\%), causes insignificant deterioration in closed-loop quality for a broad range of rescheduling frequencies, and moving horizon lengths. This can be attributed to the fact that the true gaps are significantly smaller than the estimated gaps, and also due to the feedback. Hence, in general, it can be an acceptable trade-off to not solve each open-loop problem to optimality or close to optimality. An acceptable value of OPTCR does not depend heavily on the demand pattern, but rather on the network facility and scheduling objective.

\subsection{Cross-relation between design attributes}

Now that we know how rescheduling frequency, moving horizon length and suboptimality of solution to open-loop problem affect the closed-loop quality, it is natural to ask if there is any combined effect of these design attributes. Possibly, a longer moving horizon would have already accounted for forthcoming orders, and hence, might not require frequent rescheduling in the deterministic case, where orders do not change. A shorter moving horizon, on the other hand, does not account for impending orders outside its extent and hence requires frequent rescheduling. In addition, if rescheduling is to be performed in an online fashion with limited time available for computing, then an interesting question is whether a larger problem (long moving horizon) should be solved, potentially sub-optimally, rather than a smaller problem (short moving horizon) solved to optimality. Would a faster rescheduling frequency compensate for suboptimal computations through admittance of frequent corrective feedback?

When we analyze cross relation between design attributes, we observe that a longer moving horizon, indeed, completely compensates for slower rescheduling frequency. This is because using a long moving horizon allows us to look ahead far enough to take good 
decisions early. In other words new information that enters the horizon is still far enough to take action on it now, thereby mitigating the need to reschedule frequently. This conclusion however only holds for the deterministic case, where once an order enters the moving horizon, it does not change. In the stochastic case, if the order size changes while it is within the moving horizon, rescheduling would be required.

There is also evidence suggesting that suboptimal open-loop solutions do not necessarily lead to poor closed-loop schedules due to correction through feedback. Hence, being able to reschedule frequently, even if obtaining suboptimal solutions, may have economic benefits. It is not very clear however, if a longer moving horizon is less prone to deterioration in quality than a shorter moving horizon.

\section{Case studies}

\subsection{Case study-I: Design attributes}

We compute a closed-loop schedule for PN-1 (Fig. 1) for 1-week (cost minimization) to meet a demand pattern created from tuple $(p=6, \Delta p=1, m=6, \Delta m=0)$. First, we consider $\mathrm{MH}=24, \mathrm{RF}=3$, and $\mathrm{OPTCR}=0 \%$, and obtain a closed-loop cost of $\$ 190,744$. Next, we consider $\mathrm{MH}=36, \mathrm{RF}=6$, and $\mathrm{OPTCR}=5 \%$. We use a larger OPTCR and $\mathrm{RF}=6$ to emulate the practical situation where solving a larger problem to optimality will require more time than the time available between iterations. Surprisingly, in the second simulation, we get an improved closed-loop cost of $\$ 184,947$, which is a $3.13 \%$ improvement over the first simulation. The resulting Gantt charts for both simulations are shown in Fig. 18.

Thus, we improved one design attribute, i.e. increased horizon length from 24 to 36 hours, but took a step back in two design attributes, i.e. reduced rescheduling frequency from every 3 to every 6 hours, and increased OPTCR from $0 \%$ to $5 \%$. In spite of this, we got an improved closed loop schedule. This brings up the important aspect that depending on the network facility, and particular demand pattern, some design attributes are more important than others. Even for the same network facility, with a change in demand pattern, the relative 


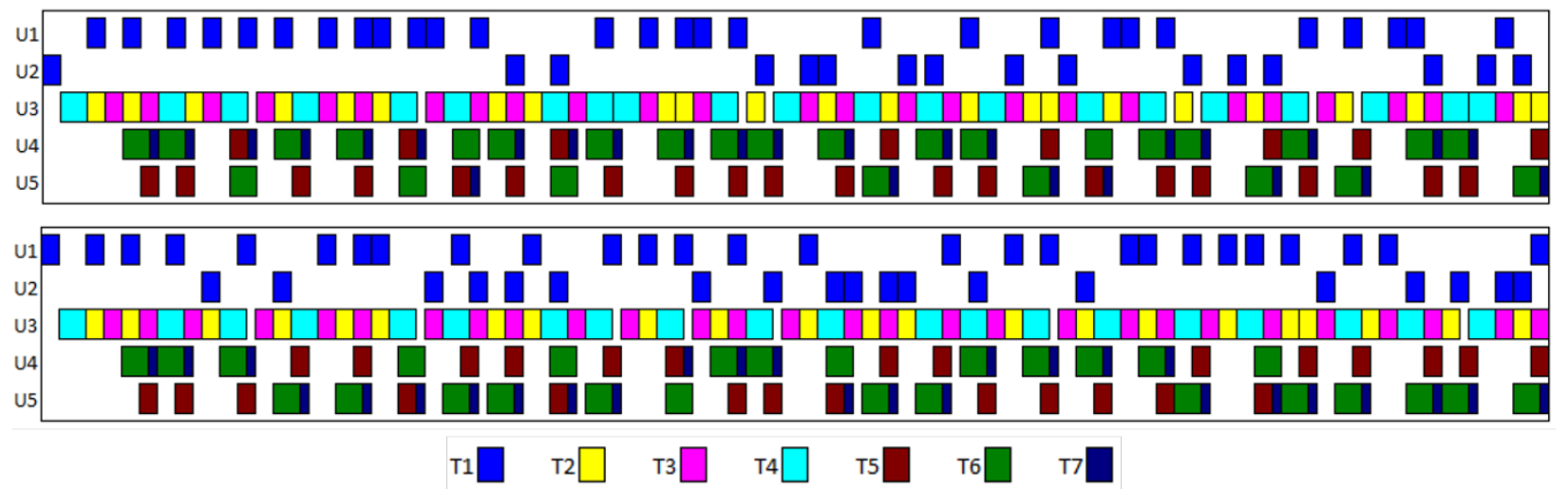

Figure 18: Gantt charts showing closed-loop schedules for $\mathrm{RF}=3, \mathrm{MH}=24, \mathrm{OPTCR}=0 \%$ (top) and $\mathrm{RF}=6$, $\mathrm{MH}=36, \mathrm{OPTCR}=5 \%$ (bottom) for a sample from demand pattern described by tuple $(p=6, \Delta p=1, m=$ $6, \Delta m=0)$. The second simulation shows an improvement of $3.13 \%$ in profit over the first simulation.

importance of design attributes can change.

In other words, we see that moving horizon length, rescheduling frequency and OPTCR work in conjunction with each other, and just considering them individually is not sufficient. Some combinations give better closed loop schedules than others; several combinations can also give equally good closed-loop schedule quality. We can choose multiple equally good combinations over others, taking into account overall closed loop solution quality, and then a specific combination among these considering implementation feasibility for a scheduling application.

\subsection{Case study-II: Instance characteristics}

In this case study we investigate how certain instance characteristics, such as process network parameters or order sizes/due-times, influence the choice of rescheduling frequency and moving horizon length.

\subsubsection{Rescheduling frequency}

We consider PN-3 (Fig. 7) with orders of size of 5 tons for each product (M2,M3) due every 4 hours (for $t \geq 8$ ), and compute a closed-loop schedule for 24-hours with $\mathrm{MH}=8$ and $\mathrm{RF}=4$ (cost minimization, zero initial inventory). We find that all orders are met on time with a closed-loop cost of $\$ 16,765$. Even if we reschedule faster, $R F=1$, we still get 
the same closed-loop schedule. This is because the 8 hour moving horizon is long enough to account for new information, and the rescheduling frequency is sufficient to act upon it to appropriately revise current decisions. However, if we now modify the process network to reduce max batch-size capacity for task $\mathrm{T} 2$ from 10 tons to 5 tons, $\mathrm{RF}=1$ results in better closed-loop performance than $\mathrm{RF}=4$. This is because, with a reduced batch-size capacity and unchanged moving horizon length (look-ahead capability), prompt actions (revisions) are needed to be able to respond to new orders. For this reduced batch-size scenario, if we use $\mathrm{MH}=12$, then $\mathrm{RF}=4$ and $\mathrm{RF}=1$ give identical closed-loop solutions. Closed-loop costs are tabulated in Table 2, and select Gantt charts are shown in Fig. 19.

Table 2: 24-hour closed-loop costs for meeting orders of 5 tons of product materials (M2,M3) due every 4 hours. Reducing $\beta_{T 2}^{M A X}$ increases the lead time for meeting orders, thus requiring faster rescheduling for myopic horizons to obtain better closed-loop solutions.

\begin{tabular}{lcccc}
\hline Look-ahead & $\mathrm{MH}$ & $\beta_{T 2}^{M A X}$ & $\mathrm{RF}=1$ & $\mathrm{RF}=4$ \\
\hline \multirow{2}{*}{ Case1-Short } & 8 & 10 & $\$ 16,765$ & $\$ 16,765$ \\
& 8 & 5 & $\$ 18,765$ & $\$ 31,764$ \\
Case2-Medium & 10 & 10 & $\$ 16,765$ & $\$ 16,765$ \\
& 10 & 5 & $\$ 18,014$ & $\$ 18,765$ \\
Case3-Long & 12 & 10 & $\$ 16,765$ & $\$ 16,765$ \\
& 12 & 5 & $\$ 18,517$ & $\$ 18,517$ \\
\hline
\end{tabular}

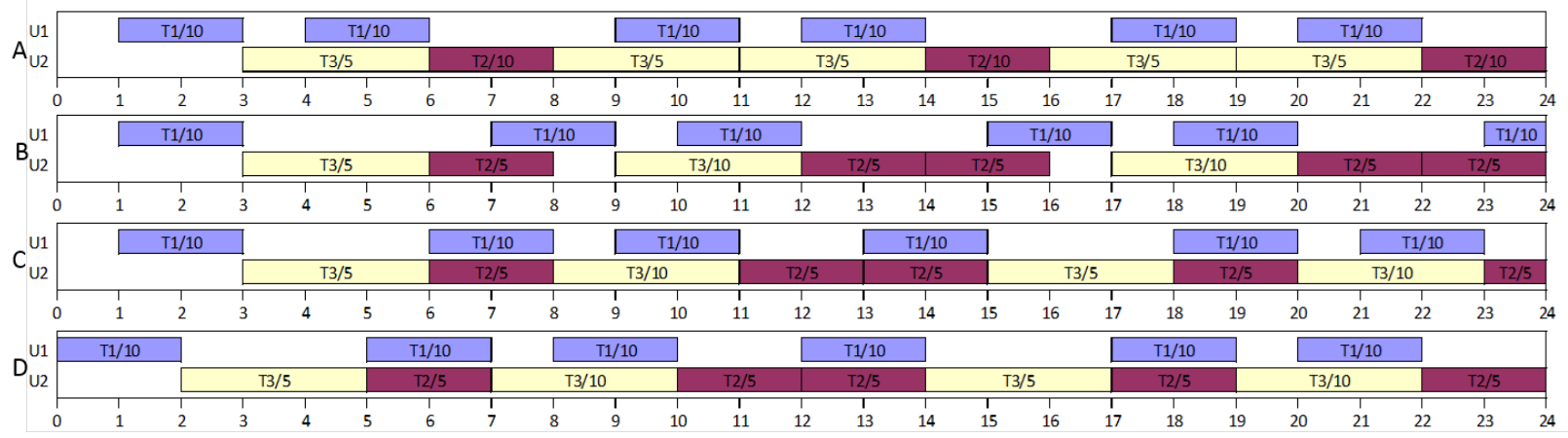

Figure 19: Orders of size 5 tons of each product material (M2,M3) are due every 4 hours $(t \geq 8)$. With zero initial inventory and cost minimzation objective, closed-loop Gantt charts for $(\mathrm{A}) \mathrm{MH}=8, \mathrm{RF}=4, \beta_{T 2}^{M A X}=10$ : Cost $\$ 16,765$; (B) $\mathrm{MH}=8, \mathrm{RF}=4, \beta_{T 2}^{M A X}=5$ : Cost $\$ 31,764$; (C) $\mathrm{MH}=8, \mathrm{RF}=1, \beta_{T 2}^{M A X}=5$ : Cost $\$ 18,765$; and (D) $\mathrm{MH}=12, \mathrm{RF}=4, \beta_{T 2}^{M A X}=5$ : Cost $\$ 18,517$.

We observe that when lead times are long, rescheduling faster is important. However, when lead times are much shorter than the moving horizon length, new orders in the latter 
part of the moving horizon do not influence current decisions, consequently, a slow rescheduling frequency suffices. The lead time to meet orders depends on the order sizes, the processing time of tasks, and as shown in this example on task batch-size capacities. We see how $\beta_{T 2}^{M A X}$, which is a process network characteristic, can influence lead times and thus the choice of rescheduling frequency. In fact, we are able to identify an upper threshold for rescheduling frequency for some of these scenarios. For example, for $\mathrm{MH}=12$ and $\beta_{T 2}^{M A X}=5, \mathrm{RF}=4$ is an upper threshold, and any faster rescheduling than this frequency does not improve the closed-loop solution any further.

In the examples discussed above, it is evident that, in the deterministic case, it is the finiteness of the moving horizon, that necessitates rescheduling. Hence finding the appropriate rescheduling frequency becomes relevant when the finite moving horizon is myopic. Next, we study the characteristics that dictate the answer to this question.

\subsubsection{Moving horizon length}

In the following set of examples, we explore some instance characteristics that govern the choice of moving horizon length. We again consider PN-3 (Fig. 7), but now with orders of 10 tons of each product (M2,M3) due every 8 hours. The network facility starts with enough inventory of intermediate M1, required by tasks T2 and T3 to meet the first order. We find a closed-loop schedule (cost minimization) for 24 hours with $\mathrm{MH}=8$ and $\mathrm{RF}=1$. The schedule incurs a cost of $\$ 11,510$ and the corresponding Gantt chart is shown in Fig. 20A. If we try using a longer moving horizon length, the closed-loop cost does not improve.

If each order size were to now be 15 tons of product M2 and 10 tons of product M3, we see that with $\mathrm{MH}=8$ we are unable to meet these larger orders on time (Cost:\$25,513, Fig. 20B). However, if we increase MH to 10 hours, these orders are also met (Cost: $\$ 21,514$, Fig. 20C). A similar effect can also be seen if the order sizes were to still be 10 tons of M2 and

M3 due every 8 hours, but if $\tau_{T 2}$ was to be 4 hours instead of 2 hours, or if $\beta_{T 2}^{M A X}$ was 5 tons instead of 10 tons (Fig. 21). Hence, the key to meet orders on time is to have moving horizon length to be greater than the time needed to meet the orders. This time depends on 


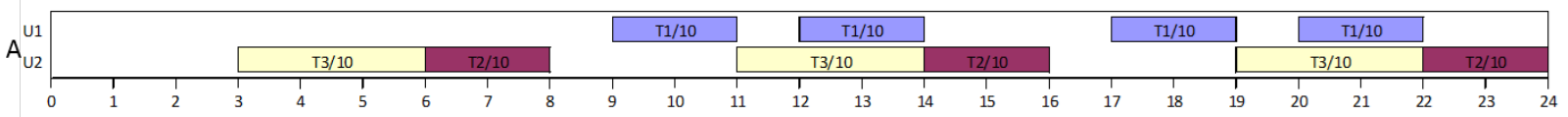

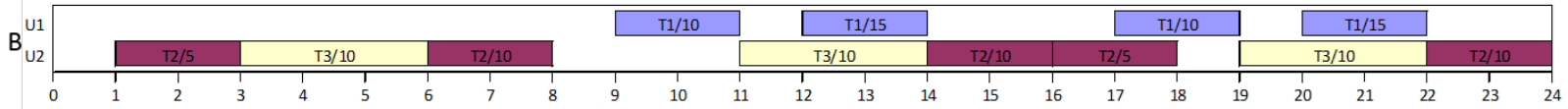

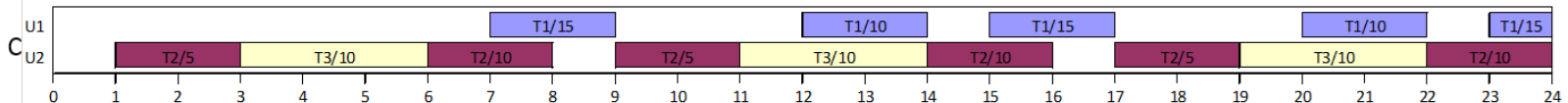

Figure 20: (A) Closed-loop schedule for PN-3 with orders of 10 tons of each product (M2,M3) due every 8 hours, with $\mathrm{MH}=8$, rescheduling every hour. Closed-loop cost is $\$ 11,510$ and it does not improve with increasing MH. (B) Orders size when increased to 15 tons of M2 and 10 tons of M3, closed-loop schedule found with $\mathrm{MH}=8$ and $\mathrm{RF}=1$, does not meet orders on time. Closed-loop cost is $\$ 25,513$. (C) For the increased order sizes, if closed-loop schedule is computed using $\mathrm{MH}=10$, all orders are met on time. Closed-loop cost is $\$ 21,514$.

the order sizes, the processing time of tasks $(\tau)$, and maximum batch-size capacities $\left(\beta^{M A X}\right)$.

When multiple small but frequent orders are due, it is not cost effective and in fact, not even feasible to run a separate batch to meet each order. Thus, big batches are executed periodically and the resulting inventory is used to meet these small orders. In order to produce enough inventory ahead of time to meet these small orders, it is required to have a long enough moving horizon, such that it covers not only the time to execute the necessary batches, but also enough orders (due-times) which would be met by these batches. For example, when small orders of 1.25 tons of each product (M2,M3) are due every hour starting at $\mathrm{t}=8$, a longer moving horizon of 12 hours rather than 8 hours, is needed to meet these orders on time (Fig. 22). We note that this demand is equivalent on a per hour basis, to 10 tons of M2,M3 due every 8 hours, but to meet these smaller but more frequent orders, a longer moving horizon $(\mathrm{MH}=12$ instead of $\mathrm{MH}=8)$ is needed.

In a complicated process network, finding a priori the time needed to meet orders is a challenging problem, since it depends on the interplay of processing times, batch-size capacities, and size and product-mix of the orders. If an estimated upper bound on the time necessary to meet orders can be found, it can be used to conservatively set moving horizon length. 

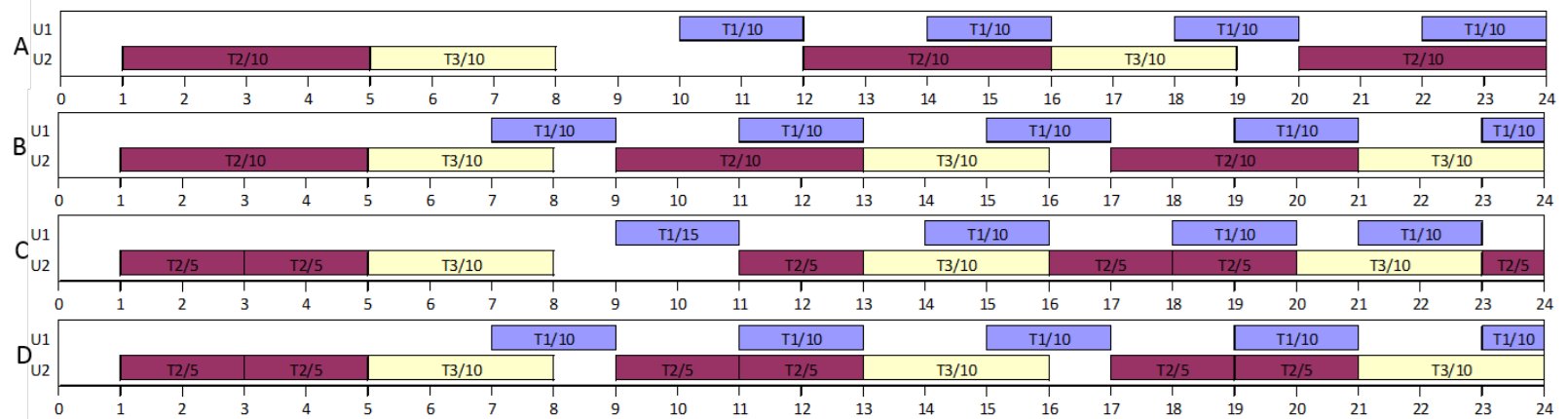

Figure 21: (A) Closed-loop schedule with cost $\$ 37,010$, when orders of 10 tons of each product (M2,M3) are due every 8 hours, found with $\mathrm{MH}=8, \mathrm{RF}=1$; Since $\tau_{T 2}=4$, the time needed to produce for each order is longer, and hence orders are not met on time. (B) Using $\mathrm{MH}=10$, all orders are met on time with closed-loop cost $\$ 13,011$. (C) Instead of increased $\tau_{T 2}$, if $\beta_{T 2}^{M A X}$ is decreased to 5 , using $\mathrm{MH}=8$, orders are not met on time and the closed-loop cost is $\$ 24,763$. (D) For $\beta_{T 2}^{M A X}=5$, using $M H=10$, all orders are met on time with closed-loop cost $\$ 17,514$.

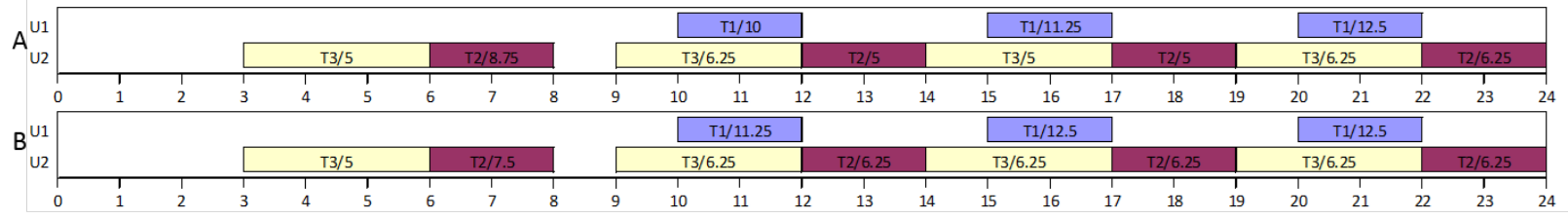

Figure 22: (A) Multiple small orders of 1.25 tons of each product M2 and M3 are due every hour starting at $\mathrm{t}=8$; On a per hour basis the demand is equivalent to if orders of 10 tons of M2,M3 were due every 8 hours; facility starts with 20 tons of intermediate M1; A closed-loop schedule with $\mathrm{MH}=8$ and $\mathrm{RF}=1$, does not meet all orders on time and costs $\$ 20,449.5$ (B) A closed-loop schedule with $\mathrm{MH}=12$, meets all orders on time and costs $\$ 18,949.5$. Longer moving horizons $(\mathrm{MH} \geq 12)$ do not lead to any further improvement.

\subsection{Case study-III: Objective function modifications and constraints}

Let us now again consider the motivating example presented in Sec. 3.1.3, in which the open-loop objective favors early sales (greedy objective). Specifically, sales at the start of the moving horizon yield twice the profit than sales at end of the moving horizon, with sale prices in between interpolated linearly. We investigate the effect of moving horizon length, rescheduling frequency, and OPTCR for profit maximization with this greedy objective. We get qualitatively identical trends for closed-loop quality as a function of rescheduling frequency and moving horizon length, as for the case with non-greedy objective (Figs. 12, 14) for all instance types. However, for suboptimal computations with greedy objective, when order due-times, and hence sales opportunities, are frequent (every 3 hours), we observe consistently that the closed-loop profit improves with increasing OPTCR (Fig. 23)! For 
lower frequency of orders (every 6 hours or 12 hours), the closed-loop profit with greedy objective does not change.

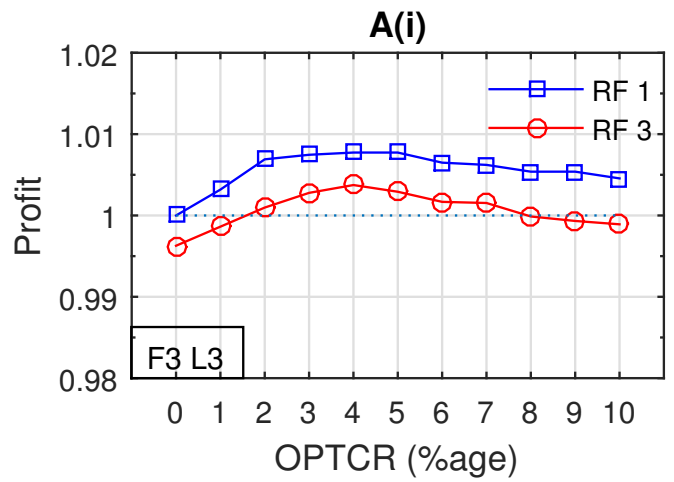

Figure 23: Effect of OPTCR on closed-loop quality for profit maximization objective. Moving horizon length of 24 hours is used. Each open-loop optimization has greedy objective. Each data point is an average profit of closed-loop schedules for 100 demand samples (pANOvA-value in supplementary information), and the profit is scaled by mean closed-loop profit corresponding to $\mathrm{OPTCR}=0 \%, \mathrm{RF}=1$.

We study this further by analyzing the instance shown in Figs. 8 and 9. The cumulative demand for material M3 over 1 week is 168 tons, hence, the minimum number of executions required for task T3, with $\beta_{T 3, U 2}^{M A X}=10$, to meet this demand is 17 . An increase in the number of executions of T3 in comparison to the minimum needed, is expected due to the finiteness of the moving horizon. A finite (myopic) moving horizon $(\mathrm{MH}=12)$ does not account for demands far ahead in future and ships out any excess inventory of M3 as early as possible (due to the greedy objective); when a new demand of M3 enters the horizon, execution of a new T3 is required to meet this demand. The greedy objective aggravates this effect by shipping early and not maintaining an appropriate inventory of M3 to meet future demand. In a suboptimal solution, early sales are reduced and excess inventory of M3 is held which is later used to meet new demand. Thus, a move that appears to be suboptimal in the current iteration (favoring inventory over sales) turns out to be a good move in the long run, leading to a better closed-loop schedule. This improvement with OPTCR though holds only till a certain threshold value (here 5\%); beyond this value the open-loop solutions cannot be repaired. For example, both T2 and T3 have a small number of executions resulting in the introduction of idle time and thereby less sales and profit. 
This myopic behavior can be corrected by, for example, adding a constraint disallowing excess-sales of M3 in the first half of the moving horizon. Since sales are allowed in the second half, excess M3 can still be produced in the first half. As shown in Fig. 24, this added constraint leads to the best possible closed-loop schedule (for both OPTCR $=0 \%$ and $\mathrm{OPTCR}=5 \%$ ) with the minimum required 17 executions of T3. In the same figure we also show the closed-loop schedule obtained using a non-greedy open-loop objective (no favoring of early sales). For this objective, although the number of executions of T3 are 18 (close to the best minimum 17), there are missed opportunities (idle times) in the schedule. These idle times can be explained by the right-shifting of tasks due to multiplicity of solutions, as discussed in the motivating example in Sec. 3.1.2.

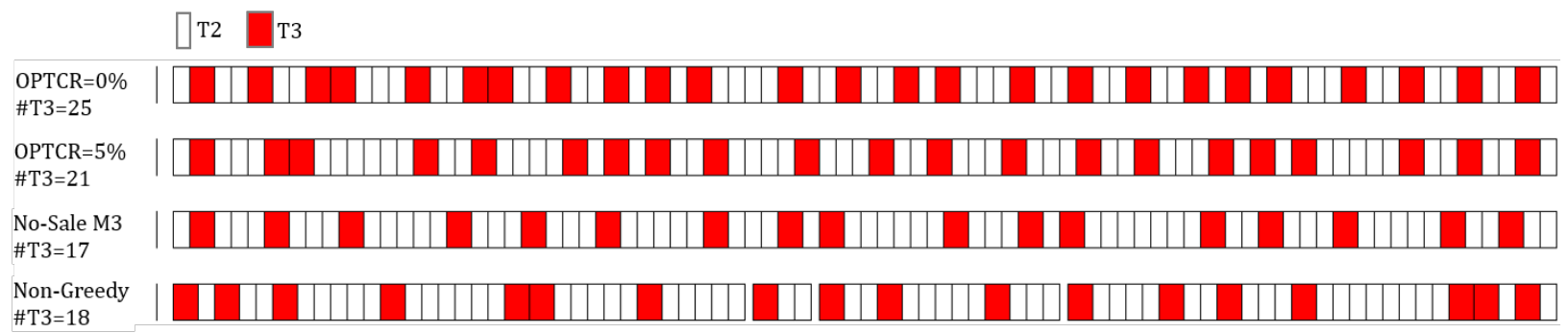

Figure 24: Closed loop schedules for unit 2; $\mathrm{MH}=12$ with OPTCR values $0 \%$ (first), $5 \%$ (second) and with added constraint (third); with OPTCR $=0 \%$, T3 is executed 25 times, while, with OPTCR $=5 \%$ T3 is executed 21 times. The added constraint (for both OPTCR $=0 \%$ and $5 \%$ ) brings down the executions of T3 to the best minimum of 17 . Non-Greedy (fourth) corresponds to $\mathrm{MH}=12, \mathrm{RF}=1$, OPTCR $=0 \%$ for open-loop objective that does not favor early sales; it has 18 executions of T3 but has idle times (missed opportunities). The Gantt charts correspond to ALG 1, 2, 5 and 7 (Table 3) respectively.

Finally, we try different ways (algorithms, ALG) to modify the open-loop problem (results presented in Table 3). In ALG 1, 3, and 4 we can see that the closed-loop profit improves with increasing moving horizon length with the best solution achieved for $\mathrm{MH}=24$. For myopic moving horizons (ALG 1, 2, and 7), the added No-Sale M3 constraint (ALG 5, 6, and 8 respectively) results in best closed-loop profits. Thus, by solving smaller open-loop problems $(\mathrm{MH}=12)$ but with the addition of a constraint we are able to get good closed-loop solutions. This is an important advantage, as we now do not have to solve larger problems $(\mathrm{MH}=24)$. However, it is interesting to see in algorithms 9 to 14 , that although the added constraint is able to improve closed-loop profit for OPTCR $\leq 5 \%$, it is not able to do so for 
Table 3: Several algorithms (ALGs) to modify the open-loop problem and their resulting closed-loop profits. Checkmark $(\checkmark)$ in Obj column indicates greedy objective. NoSale-M3 refers to the addition of a constraint to not sell any excess M3 in first half of the moving horizon.

\begin{tabular}{|c|c|c|c|c|c|c|c|c|}
\hline & $\mathrm{RF}$ & $\mathrm{MH}$ & OPTCR & Greedy-Obj & NoSale-M3 & T3-Executions & $\operatorname{Profit}(\$)$ & Profit (Scaled) \\
\hline ALG1 & 1 & 12 & $0 \%$ & $\checkmark$ & & 25 & 3652 & 0.926 \\
\hline ALG2 & 1 & 12 & $5 \%$ & $\checkmark$ & & 21 & 3849 & 0.976 \\
\hline ALG3 & 1 & 18 & $0 \%$ & $\checkmark$ & & 21 & 3847 & 0.975 \\
\hline ALG4 & 1 & 24 & $0 \%$ & $\checkmark$ & & 17 & 3945 & 1.000 \\
\hline ALG5 & 1 & 12 & $0 \%$ & $\checkmark$ & $\checkmark$ & 17 & 3949 & 1.001 \\
\hline ALG6 & 1 & 12 & $5 \%$ & $\checkmark$ & $\checkmark$ & 17 & 3947 & 1.001 \\
\hline ALG7 & 1 & 12 & $0 \%$ & & & 18 & 3849 & 0.976 \\
\hline ALG8 & 1 & 12 & $0 \%$ & & $\checkmark$ & 17 & 3947 & 1.001 \\
\hline ALG9 & 3 & 12 & $0 \%$ & $\checkmark$ & & 25 & 3652 & 0.926 \\
\hline ALG10 & 3 & 12 & $0 \%$ & $\checkmark$ & $\checkmark$ & 17 & 3948 & 1.001 \\
\hline ALG11 & 3 & 12 & $5 \%$ & $\checkmark$ & & 24 & 3652 & 0.926 \\
\hline ALG12 & 3 & 12 & $5 \%$ & $\checkmark$ & $\checkmark$ & 17 & 3948 & 1.001 \\
\hline ALG13 & 3 & 12 & $10 \%$ & $\checkmark$ & & 21 & 3849 & 0.976 \\
\hline ALG14 & 3 & 12 & $10 \%$ & $\checkmark$ & $\checkmark$ & 17 & 3849 & 0.976 \\
\hline
\end{tabular}

OPTCR $=10 \%$. This is because in ALG 13, the bad performance is due to a fundamentally bad schedule (idle times), and not due to selling excess M3 early. Hence, an added constraint to not sell M3 early (ALG 14), although decreases the number of executions of T3, it is not able to correct for the idle times present in the schedule found using ALG 13. This example thus shows how adding constraints to open-loop problem is a powerful and a much more effective way to improve closed-loop performance than modifications to objective function or closed-loop design attributes (RF.MH.OPTCR); however, finding the appropriate constraints for each situation is a challenge and requires further investigation.

\section{Conclusions}

We presented a framework for the analysis of closed-loop schedules. First, we showed that open-loop and closed-loop scheduling are two different problems, even in the deterministic case when no uncertainty is present. Applying methods to improve solution to the openloop problem, does not necessarily translate to good solutions for the closed-loop problem. Second, we found that it is important to reschedule periodically, even when there are no 
"trigger" events, something that is in contrast with the current approaches to rescheduling. Third, we showed that suboptimalities in the re-optimizations do not "accumulate", but instead, are corrected through repetitive revisions due to feedback. Fourth, we studied how rescheduling frequency, moving horizon length and suboptimal solutions of open-loop problem affect the quality of closed-loop schedules, and found that there exist certain threshold values, operating outside of which leads to bad closed-loop solutions. These thresholds, which depend on characteristics of the network facility, and the demand pattern, can be utilized to appropriately choose the online scheduling design attributes (MH, RF, and OPTCR). However, we also found that there is a cross-relation between these design attributes, and hence, we should choose an appropriate value for all three in conjunction. Lastly, we explored objective function modifications and addition of constraints to the open-loop problem as effective methods to improve closed-loop performance. Notably, we showed that adding constraints can possibly lead to lower quality open-loop solutions, but can ultimately result in higher quality closed-loop (implemented) solutions. However, further work is needed to find appropriate constraints that could improve closed-loop solution in the general case. The scope of this work was limited to deterministic rescheduling, since as shown through the motivating examples, the deterministic case itself is laden with several "paradoxes". However, uncertainty is also a major concern, thus studying rescheduling under uncertainty is an interesting future direction. Finally, we would like to note that the framework and analysis presented in this paper are applicable to all discrete-time scheduling models based on STN as well as RTN representations.

\section{Acknowledgments}

The authors would like to acknowledge support from the National Science Foundation under grants CMMI-1334933 and CBET-1264096, as well as the Petroleum Research Fund under grant 53313-ND9. CTM would like to thank Dr. James Rawlings for fruitful discussions on model predictive control and, specifically, on the role of feedback. 


\title{
Nomenclature
}

\author{
Indices/sets \\ $i \in \mathbf{I} \quad$ tasks \\ $j \in \mathbf{J} \quad$ units (equipment) \\ $k \in \mathbf{K} \quad$ materials \\ $t \in \mathbf{T} \quad$ time-periods/points \\ $l \in \mathbf{L} \quad$ orders \\ $\mathbf{I} \supseteq \mathbf{I}_{j} \quad$ tasks that can be carried out in unit $j$ \\ $\mathbf{I} \supseteq \mathbf{I}_{k}^{+} \quad$ tasks producing material $k$ \\ $\mathbf{I} \supseteq \mathbf{I}_{k}^{-} \quad$ tasks consuming material $k$ \\ $\mathbf{K} \supseteq \mathbf{K}^{F} \quad$ feed (raw) materials \\ $\mathbf{K} \supseteq \mathbf{K}^{I} \quad$ intermediates \\ $\mathbf{K} \supseteq \mathbf{K}^{P} \quad$ final products
}

Parameters

$\beta_{i j}^{M I N} / \beta_{i j}^{M A X}$

$\min / \max$ capacity on batch-size of task $i$ executed on unit $j$

$\delta$

length of time-period

$\eta$

scheduling horizon

$\sigma$

Open-loop iteration number

$\rho_{i k}$

conversion coefficient

$\tau_{i}$

processing time of (batch) task $i$

$\zeta_{k t}$

incoming shipment of material $k$ at time $t$

$\xi_{k t}$

demand for material $k$ at time $t$

$\alpha_{i j}^{F}$

$\alpha_{i j}^{P}$

$\pi_{k}$

$\pi_{k}^{I N V}$

fixed cost of task $i$ on unit $j$

proportional cost of task $i$ on unit $j$

selling price of material $k$

inventory cost of material $k$ 
$\pi_{k}^{B O}$

Variables

$\begin{array}{ll}W_{i j t} \in\{0,1\} & 1 \text { if (batch of) task } i \text { starts at time point } t \text { in unit } j \\ B_{i j t} & \text { batch-size of task } i \text { in unit } j \\ S_{k t} & \text { inventory level of material } k \text { during period }(t-1, t] \\ B O_{k t} & \text { backlog level of material } k \text { during period }(t-1, t] \\ V_{k t}^{D} & \text { outgoing shipment to meet demand for material } k \text { at time } t \\ V_{k t}^{S} & \text { outgoing shipment as excess-sales of material } k \text { at time } t \\ V_{k t} & \text { total outgoing shipment of material } k \text { at time } t \\ \bar{W}_{i j t}^{n} \in\{0,1\} & \text { lifted assignment variables } \\ \bar{B}_{i j t}^{n} & \text { lifted batch-size variables }\end{array}$

Abbreviations

OBJ

PN

D

S

$\mathrm{RF}$

$\mathrm{MH}$

OPTCR

pANOVA

$\mathrm{r}_{\text {Spear }}$

$\mathrm{p}_{\text {Spear }}$

Demand pattern attributes backlog cost of material $k$
Open-loop objective function

Process Network

Demand pattern

Demand sample

Rescheduling frequency

Moving horizon length

Optimization termination criterion based on relative estimated gap

Statistical credibility of null hypothesis in ANOVA analysis

Spearman correlation coefficient

p-value associated with Spearman correlation coefficient

Mean time-period between orders 
$\Delta p$

$m$

$\Delta m$

\author{
Variation of order due-times around the mean positions \\ Mean magnitude of demand of each product within an order \\ Variation in magnitude around mean for every order
}

\section{References}

Balasubramanian, J., Grossmann, I., 2004. Approximation to multistage stochastic optimization in multiperiod batch plant scheduling under demand uncertainty. Industrial \& Engineering Chemistry Research 43 (14), 3695-3713.

Balasubramanian, J., Grossmann, I. E., 2003. Scheduling optimization under uncertainty-an alternative approach. Computers \& Chemical Engineering 27 (4), 469-490.

Bassett, M. H., Pekny, J. F., Reklaitis, G. V., 1996. Decomposition techniques for the solution of large-scale scheduling problems. AIChE Journal 42 (12), 3373-3387.

Bassett, M. H., Pekny, J. F., Reklaitis, G. V., 1997. Using detailed scheduling to obtain realistic operating policies for a batch processing facility. Industrial \& Engineering Chemistry Research 36 (5), 1717-1726.

Bonfill, A., Bagajewicz, M., Espuña, A., Puigjaner, L., 2004. Risk management in the scheduling of batch plants under uncertain market demand. Industrial \& engineering chemistry research $43(3), 741-750$.

Bonfill, A., Espuña, A., Puigjaner, L., 2005. Addressing robustness in scheduling batch processes with uncertain operation times. Industrial \& engineering chemistry research $44(5), 1524-1534$.

Cott, B., Macchietto, S., 1989. Minimizing the effects of batch process variability using online schedule modification. Computers \& Chemical Engineering 13 (1), 105-113. 
Cui, J., Engell, S., 2010. Medium-term planning of a multiproduct batch plant under evolving multi-period multi-uncertainty by means of a moving horizon strategy. Computers \& Chemical Engineering 34 (5), 598-619.

Dirkse, S., Ferris, M. C., Ramakrishnan, J., 2014. GDXMRW-Interfacing GAMS and MATLAB, GAMS Documentation/Tools/Data Exchange, Accessed February 8, 2016. URL http: //www.gams.com/help/

Du, J., Park, J., Harjunkoski, I., Baldea, M., 2015. A time scale-bridging approach for integrating production scheduling and process control. Computers \& Chemical Engineering 79, 59-69.

Harjunkoski, I., Maravelias, C. T., Bongers, P., Castro, P. M., Engell, S., Grossmann, I. E., Hooker, J., Méndez, C., Sand, G., Wassick, J., 2014. Scope for industrial applications of production scheduling models and solution methods. Computers \& Chemical Engineering $62,161-193$.

Honkomp, S., Mockus, L., Reklaitis, G., 1999. A framework for schedule evaluation with processing uncertainty. Computers \& Chemical Engineering 23 (4), 595-609.

HTCondor 8.5.0, 2015. Department of Computer Sciences, University of Wisconsin-Madison, Accessed February 8, 2016.

URL https://research.cs.wisc.edu/htcondor/

Huercio, A., Espuna, A., Puigjaner, L., 1995. Incorporating on-line scheduling strategies in integrated batch production control. Computers \& Chemical Engineering 19, 609-614.

Janak, S. L., Floudas, C. A., Kallrath, J., Vormbrock, N., 2006. Production scheduling of a large-scale industrial batch plant. ii. reactive scheduling. Industrial \& Engineering Chemistry Research 45 (25), 8253-8269. 
Janak, S. L., Lin, X., Floudas, C. A., 2007. A new robust optimization approach for scheduling under uncertainty: Ii. uncertainty with known probability distribution. Computers \& Chemical Engineering 31 (3), 171-195.

Kanakamedala, K. B., Reklaitis, G. V., Venkatasubramanian, V., 1994. Reactive schedule modification in multipurpose batch chemical plants. Industrial \& Engineering Chemistry Research 33 (1), 77-90.

Kazan, O., Nagi, R., Rump, C. M., 2000. New lot-sizing formulations for less nervous production schedules. Computers \& Operations Research 27 (13), 1325-1345.

Kelly, J., Mann, J., 2003a. Crude oil blend scheduling optimization: an application with multimillion dollar benefits-part 1: Process/plant optimization. Hydrocarbon Processing $82(6), 47-53$.

Kelly, J., Mann, J., 2003b. Crude oil blend scheduling optimization: an application with multimillion dollar benefits. part 2. Hydrocarbon processing 82 (7), 72-79.

Kelly, J. D., Zyngier, D., 2008. Hierarchical decomposition heuristic for scheduling: Coordinated reasoning for decentralized and distributed decision-making problems. Computers \& Chemical Engineering 32 (11), 2684-2705.

Kondili, E., Pantelides, C., Sargent, R., 1993. A general algorithm for short-term scheduling of batch operationsi. milp formulation. Computers \& Chemical Engineering 17 (2), 211227.

Kopanos, G. M., Capon-Garcia, E., Espuna, A., Puigjaner, L., 2008. Costs for rescheduling actions: A critical issue for reducing the gap between scheduling theory and practice. Industrial \& Engineering Chemistry Research 47 (22), 8785-8795.

Kopanos, G. M., Pistikopoulos, E. N., 2014. Reactive scheduling by a multiparametric pro- 
gramming rolling horizon framework: a case of a network of combined heat and power units. Industrial \& Engineering Chemistry Research 53 (11), 4366-4386.

Li, Z., Ierapetritou, M., 2008a. Process scheduling under uncertainty: Review and challenges. Computers \& Chemical Engineering 32 (4), 715-727.

Li, Z., Ierapetritou, M. G., 2008b. Reactive scheduling using parametric programming. AIChE journal 54 (10), 2610-2623.

Lin, X., Janak, S. L., Floudas, C. A., 2004. A new robust optimization approach for scheduling under uncertainty:: I. bounded uncertainty. Computers \& Chemical Engineering 28 (6), 1069-1085.

Lindholm, A., Giselsson, P., Quttineh, N.-H., Lidestam, H., Johnsson, C., Forsman, K., 2013. Production scheduling in the process industry. In: 22nd International Conference on Production Research.

Maravelias, C. T., 2012. General framework and modeling approach classification for chemical production scheduling. AIChE Journal 58 (6), 1812-1828.

Méndez, C. A., Cerdá, J., 2003. Dynamic scheduling in multiproduct batch plants. Computers \& Chemical Engineering 27 (8), 1247-1259.

Méndez, C. A., Cerdá, J., Grossmann, I. E., Harjunkoski, I., Fahl, M., 2006. State-of-the-art review of optimization methods for short-term scheduling of batch processes. Computers \& Chemical Engineering 30 (6), 913-946.

Merchan, A. F., Maravelias, C. T., 2016. Preprocessing and tightening methods for timeindexed MIP chemical production scheduling models. Computers \& Chemical Engineering $84,516-535$.

Munawar, S., Gudi, R., 2005. A multilevel, control-theoretic framework for integration of 
planning, scheduling, and rescheduling. Industrial \& Engineering Chemistry Research 44 (11), 4001-4021.

Nie, Y., Biegler, L. T., Wassick, J. M., Villa, C. M., 2014. Extended discrete-time resource task network formulation for the reactive scheduling of a mixed batch/continuous process. Industrial \& Engineering Chemistry Research 53 (44), 17112-17123.

Novas, J. M., Henning, G. P., 2010. Reactive scheduling framework based on domain knowledge and constraint programming. Computers \& Chemical Engineering 34 (12), 2129-2148.

Novas, J. M., Henning, G. P., 2012. A comprehensive constraint programming approach for the rolling horizon-based scheduling of automated wet-etch stations. Computers \& Chemical Engineering 42, 189-205.

Pantelides, C. C., 1994. Unified frameworks for optimal process planning and scheduling. In: Proceedings on the second conference on foundations of computer aided operations. Cache Publications New York, pp. 253-274.

Petrovic, D., Duenas, A., 2006. A fuzzy logic based production scheduling/rescheduling in the presence of uncertain disruptions. Fuzzy sets and systems 157 (16), 2273-2285.

Rawlings, J. B., Mayne, D. Q., 2009. Model predictive control: Theory and design. Nob Hill Pub.

Relvas, S., Matos, H. A., Barbosa-Póvoa, A. P. F., Fialho, J., 2007. Reactive scheduling framework for a multiproduct pipeline with inventory management. Industrial \& Engineering Chemistry Research 46 (17), 5659-5672.

Rodrigues, M., Gimeno, L., Passos, C., Campos, M., 1996. Reactive scheduling approach for multipurpose chemical batch plants. Computers \& Chemical Engineering 20, S1215-S1220.

Ryu, J.-h., Dua, V., Pistikopoulos, E. N., 2007. Proactive scheduling under uncertainty: a 
parametric optimization approach. Industrial \& Engineering Chemistry Research 46 (24), $8044-8049$.

Sand, G., Engell, S., 2004. Modeling and solving real-time scheduling problems by stochastic integer programming. Computers \& Chemical Engineering 28 (6), 1087-1103.

Shah, N., Pantelides, C., Sargent, R., 1993. A general algorithm for short-term scheduling of batch operationsii. computational issues. Computers \& Chemical Engineering 17 (2), 229-244.

Shobrys, D. E., White, D. C., 2002. Planning, scheduling and control systems: why cannot they work together. Computers \& Chemical engineering 26 (2), 149-160.

Subrahmanyam, S., Kudva, G. K., Bassett, M. H., Pekny, J. F., 1996. Application of distributed computing to batch plant design and scheduling. AIChE journal 42 (6), 16481661.

Subramanian, K., Maravelias, C. T., Rawlings, J. B., 2012. A state-space model for chemical production scheduling. Computers \& Chemical Engineering 47, 97-110.

Subramanian, K., Rawlings, J. B., Maravelias, C. T., 2014. Economic model predictive control for inventory management in supply chains. Computers \& Chemical Engineering $64,71-80$.

Sundaramoorthy, A., Maravelias, C. T., 2011. Computational study of network-based mixedinteger programming approaches for chemical production scheduling. Industrial \& Engineering Chemistry Research 50 (9), 5023-5040.

Velez, S., Maravelias, C. T., 2013a. A branch-and-bound algorithm for the solution of chemical production scheduling mip models using parallel computing. Computers \& Chemical Engineering 55, 28-39. 
Velez, S., Maravelias, C. T., 2013b. Multiple and nonuniform time grids in discrete-time mip models for chemical production scheduling. Computers \& Chemical Engineering 53, $70-85$.

Velez, S., Maravelias, C. T., 2013c. Reformulations and branching methods for mixed-integer programming chemical production scheduling models. Industrial \& Engineering Chemistry Research 52 (10), 3832-3841.

Velez, S., Maravelias, C. T., 2015. Theoretical framework for formulating mip scheduling models with multiple and non-uniform discrete-time grids. Computers \& Chemical Engineering $72,233-254$.

Velez, S., Sundaramoorthy, A., Maravelias, C. T., 2013. Valid inequalities based on demand propagation for chemical production scheduling MIP models. AIChE Journal 59 (3), 872887.

Vieira, G. E., Herrmann, J. W., Lin, E., 2003. Rescheduling manufacturing systems: a framework of strategies, policies, and methods. Journal of scheduling 6 (1), 39-62.

Vin, J. P., Ierapetritou, M. G., 2000. A new approach for efficient rescheduling of multiproduct batch plants. Industrial \& Engineering Chemistry Research 39 (11), 4228-4238.

Wang, J., 2004. A fuzzy robust scheduling approach for product development projects. European Journal of Operational Research 152 (1), 180-194.

Wolsey, L. A., 1998. Integer programming. Vol. 42. Wiley New York.

Wonnacott, T. H., Wonnacott, R. J., 1972. Introductory statistics. Vol. 19690. John Wiley and Sons.

Yee, K., Shah, N., 1998. Improving the efficiency of discrete time scheduling formulation. Computers \& chemical engineering 22, S403-S410. 
Yu, C.-S., Li, H.-L., 2000. A robust optimization model for stochastic logistic problems. International Journal of Production Economics 64 (1), 385-397.

Zhuge, J., Ierapetritou, M. G., 2012. Integration of scheduling and control with closed loop implementation. Industrial \& Engineering Chemistry Research 51 (25), 8550-8565. 